\title{
Article
}

\section{Deep Proteomic Analysis on Biobanked Paraffine-Archived Melanoma with Prognostic/Predictive Biomarker Read-Out}

\author{
Leticia Szadai ${ }^{1, *, \dagger}$, Erika Velasquez ${ }^{2, \dagger}$, Beáta Szeitz $^{3 \oplus}$, Natália Pinto de Almeida ${ }^{4,5}{ }^{\oplus}$, Gilberto Domont ${ }^{5}$, \\ Lazaro Hiram Betancourt ${ }^{6}$, Jeovanis Gil ${ }^{6}{ }^{\circ}$, Matilda Marko-Varga ${ }^{4}$, Henriett Oskolas ${ }^{6}$, Ágnes Judit Jánosi ${ }^{1}$, \\ Maria del Carmen Boyano-Adánez ${ }^{7}$, Lajos Kemény ${ }^{1,8}{ }^{(0}$, Bo Baldetorp ${ }^{6}$, Johan Malm ${ }^{2}{ }^{\mathbb{D}}$, Peter Horvatovich ${ }^{9}{ }^{\mathbb{D}}$,

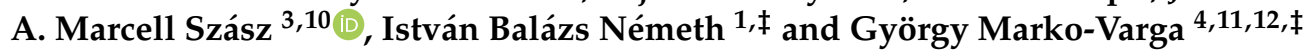

1 Department of Dermatology and Allergology, University of Szeged, 6720 Szeged, Hungary; janosi.agi97@gmail.com (Á.J.J.); kemeny.lajos@med.u-szeged.hu (L.K.); nemeth.istvan.balazs@med.u-szeged.hu (I.B.N.)

2 Section for Clinical Chemistry, Department of Translational Medicine, Lund University, Skåne University Hospital Malmö, 20502 Malmö, Sweden; erika.velasquez@med.lu.se (E.V.); johan.malm@med.lu.se (J.M.)

3 Department of Internal Medicine and Oncology, Semmelweis University, 1083 Budapest, Hungary; szeitz.beata@phd.semmelweis.hu (B.S.); szasz.attila_marcell@med.semmelweis-univ.hu (A.M.S.)

4 Clinical Protein Science \& Imaging, Biomedical Centre, Department of Biomedical Engineering, Lund University, BMC D13, 22184 Lund, Sweden; nataliapalmeida@pos.iq.ufrj.br (N.P.d.A.); matilda.marko_varga@bme.lth.se (M.M.-V.); gyorgy.marko-varga@bme.lth.se (G.M.-V.)

5 Chemistry Institute Federal, University of Rio de Janeiro, Rio de Janiero 21941-901, Brazil; gilberto@iq.ufrj.br

6 Division of Oncology, Department of Clinical Sciences Lund, Lund University, 22185 Lund, Sweden; lazaro.betancour@@med.lu.se (L.H.B.); jeovanis.gil_valdes@med.lu.se (J.G.);

updates

Citation: Szadai, L.; Velasquez, E.; Szeitz, B.; Almeida, N.P.d.; Domont, G.; Betancourt, L.H.; Gil, J.; Marko-Varga, M.; Oskolas, H.; Jánosi, Á.J.; et al. Deep Proteomic Analysis on Biobanked Paraffine-Archived Melanoma with Prognostic/Predictive Biomarker Read-Out. Cancers 2021, 13, 6105. https://doi.org/10.3390/cancers 13236105

Academic Editor: David Wong

Received: 23 October 2021

Accepted: 29 November 2021

Published: 3 December 2021

Publisher's Note: MDPI stays neutral with regard to jurisdictional claims in published maps and institutional affiliations.

Copyright: (C) 2021 by the authors. Licensee MDPI, Basel, Switzerland. This article is an open access article distributed under the terms and conditions of the Creative Commons Attribution (CC BY) license (https:/ / creativecommons.org/licenses/by/ $4.0 /)$. henriett.kovacs-oskolas@med.lu.se (H.O.); bo.baldetrop@med.lu.se (B.B.)

7 Department of Systems Biology, Faculty of Medicine and Health Sciences, University of Alcala de Henares, 28801 Alcalá de Henares, Madrid, Spain; carmen.boyano@uah.es

8 HCEMM-USZ Skin Research Group, University of Szeged, 6720 Szeged, Hungary

9 Department of Analytical Biochemistry, Faculty of Science and Engineering, University of Groningen, 9712 CP Groningen, The Netherlands; p.l.horvatovich@rug.nl

10 Department of Bioinformatics, Semmelweis University, 1094 Budapest, Hungary

11 Chemical Genomics Global Research Lab, Department of Biotechnology, College of Life Science and Biotechnology, Yonsei University, Seoul 03722, Korea

12 Department of Surgery, Tokyo Medical University, Tokyo 160-8402, Japan

* Correspondence: szadai.leticia@med.u-szeged.hu

+ These two authors contributed equally to this work as co-first authors.

$\ddagger \quad$ These two authors contributed equally to this work as co-last authors.

Simple Summary: Malignant melanoma is one of the most aggressive cancer types among the solid tumors; therefore, more clinically applicable protein biomarkers predicting survival and therapy response have mandatory importance, impacting patient treatment. The aim of the study was to discover new proteins in biobanked FFPE samples that relate to progression-free survival and response to targeted- and immuno-therapies in patients with melanoma. Protein expressions were detected and quantified by high-resolution mass spectrometry and were integrated with the clinical data and in-depth histopathology characterization. Sample groups with distinct protein expression profiles were connected to longer and shorter survival as well as other clinicopathologic features. In addition, key regulating proteins were assigned, as predictive of progression-free survival in immuno- and/or targeted therapy. Some of the proteins exhibited functionally important correlations to progression and therapy response, which ultimately contributes to a better understanding of melanoma pathology.

Abstract: The discovery of novel protein biomarkers in melanoma is crucial. Our introduction of formalin-fixed paraffin-embedded (FFPE) tumor protocol provides new opportunities to understand the progression of melanoma and open the possibility to screen thousands of FFPE samples deposited in tumor biobanks and available at hospital pathology departments. In our retrospective biobank pilot study, 90 FFPE samples from 77 patients were processed. Protein quantitation was performed by high-resolution mass spectrometry and validated by histopathologic analysis. The global protein 
expression formed six sample clusters. Proteins such as TRAF6 and ARMC10 were upregulated in clusters with enrichment for shorter survival, and proteins such as AIFI1 were upregulated in clusters with enrichment for longer survival. The cohort's heterogeneity was addressed by comparing primary and metastasis samples, as well comparing clinical stages. Within immunotherapy and targeted therapy subgroups, the upregulation of the VEGFA-VEGFR2 pathway, RNA splicing, increased activity of immune cells, extracellular matrix, and metabolic pathways were positively associated with patient outcome. To summarize, we were able to (i) link global protein expression profiles to survival, and they proved to be an independent prognostic indicator, as well as (ii) identify proteins that are potential predictors of a patient's response to immunotherapy and targeted therapy, suggesting new opportunities for precision medicine developments.

Keywords: metastatic melanoma; immunotherapy and targeted therapy responder; prognostic and predictive biomarkers; protein expression pattern in long and short survival; proteomics

\section{Introduction}

Malignant melanoma is the most metastatic human cancer among all tumor types [1,2] and was responsible for more than 50,000 deaths worldwide in 2020 [3].

The incidence of melanoma is anticipated to increase 3\% annually at least until 2022 in Norway, Sweden, the UK and the US [4].

While the primary prevention and patient education (e.g., patient factors: knowledge of the disease, educational level, patient-doctor relationship) are better than they were decades ago, the unpredictable behavior of the disease and the maintenance of a proper therapy regimen is still a struggle of the healthcare systems.

Nowadays, the first- and second-line therapy of disseminated melanoma is based on the kinase and immune checkpoint inhibitor approaches. The kinase inhibitor therapies, such as BRAF inhibitors, combined with MEK inhibitors, achieve a dramatic tumor response rate in a short period with the prolongation of the progression-free survival (PFS) and the overall survival (OS). The immune checkpoint inhibitors, such as anti-PD-1, PD-L1 and CTLA4 agents, have a prolonged effect both on the melanoma niche and on the innocent immune cells [5]. Due to the molecular mechanisms of therapy resistance and toxicity, the relapse of the disease is a common phenomenon during the treatment. Even though the effect of targeted and immunotherapy extend the objective response rate of metastatic melanoma patients to approximately 50-70\% [4,6], there are no proper predictive biomarkers or tools with high sensitivity and specificity values to predict ad hoc therapy response and distinguish among biological therapies.

Currently, at the genetic level, the mutations of driver oncogenic genes such as BRAF, NRAS, KIT, PTEN, TP53 and NF1 are considered as the mainstay of melanoma development. Interestingly, a previous study revealed that the mutated proteins of the aforementioned genes have an effect on survival [7] and may have an impact on further therapy response.

Moving forward, multiple studies demonstrated the molecular mechanisms behind the new therapies [8-10]. In a recent trial, the melanoma patients treated with anti-PD-L1 therapy showed responsiveness with the overexpression of IFN-gamma and IRE genes [1,11].

Furthermore, according to the Genomic Classification of Cutaneous Melanoma [12], PTEN mutations, PD-1, PD-L1 and MITF genes were observed in significantly higher copy numbers in BRAF-subtype melanomas defined with the presence of hot-spot mutations; thus, these genetic alterations and the aforementioned immune mechanisms explicate the importance of applied immunotherapies or the need for predictive markers regarding therapy choice and survival.

This retrospective pilot study is based on a previous analysis that could establish a high-throughput proteomic and phosphoproteomic dataset on formalin-fixed, paraffinembedded (FFPE) tumor samples compared to analysis of fresh frozen tumor (FFT) samples 
for the first time. A total of 7629 proteins and 12,659 phosphopeptides were identified, including the phospho-site quantification of the BRAF-MEK-ERK pathway [13].

In this study, we have conducted a comprehensive proteomic analysis on the FFPE melanoma samples to represent the potential molecular changes between samples, address the intratumoral heterogeneity on histopathology validation and detect proteins predictive of therapy outcome and survival. In the pilot study, 90 samples from 77 patients were involved and analyzed for both histopathologic features and for global proteomic analysis (Figure 1). We detected six groups of samples based on unsupervised hierarchical protein clustering and correlated the clusters with clinical and histopathological parameters. We also examined proteomic differences between primary and metastasis samples and identified proteins differentially expressed between the clinical stages. Additionally, protein expressions were evaluated based on whether they represent potential predictive markers for immuno/targeted therapy response. Our study aimed to address the following question: can proteomics provide us with new potential biomarkers or dysregulated pathways that are predictive of therapy response?

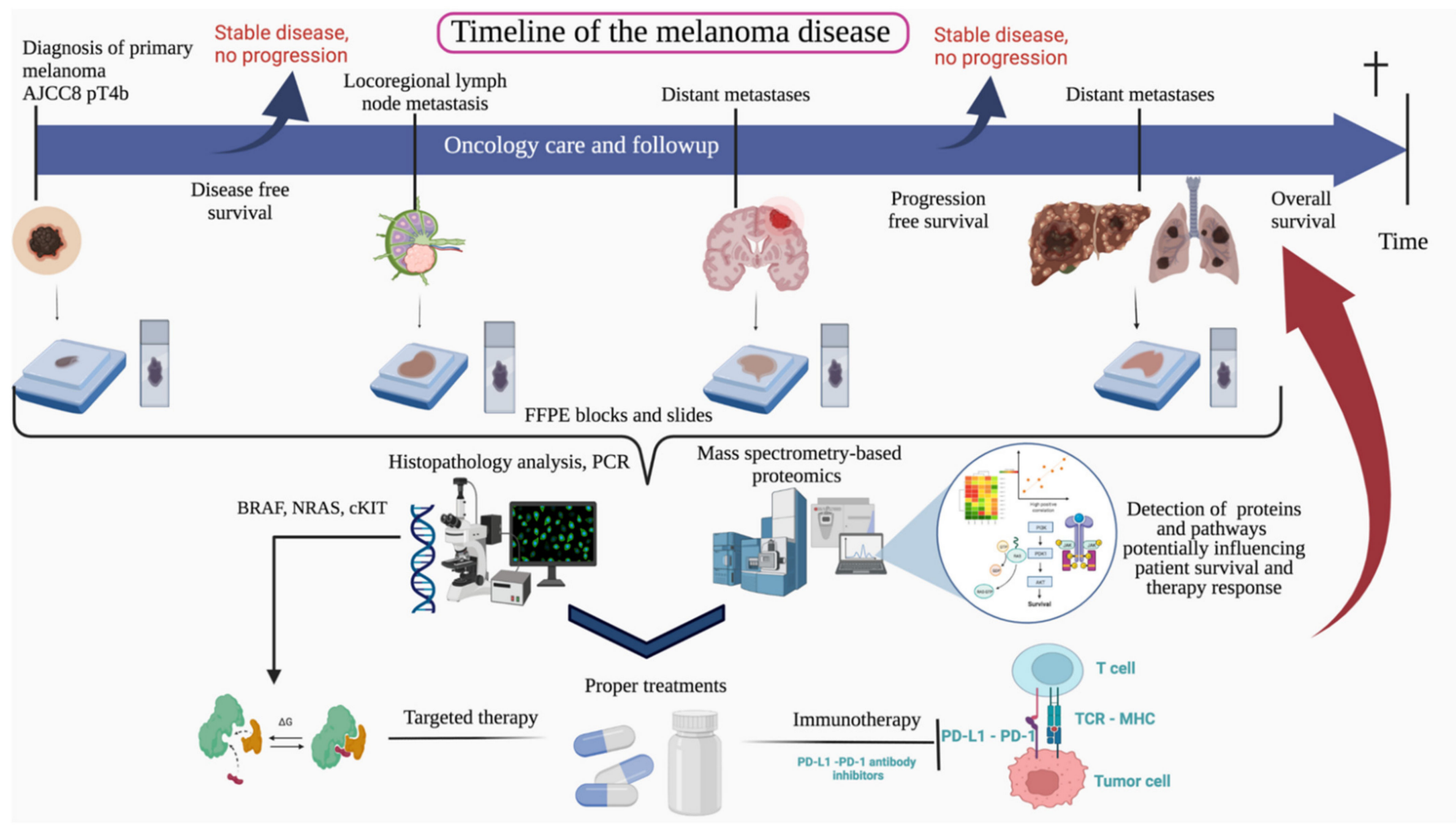

Figure 1. The overview of our study. Scheme illustrating the general working process of our study, showing the timeline of the melanoma disease, from the tumor diagnosis, through progression until the appearance of the distant metastases. This study included 90 FFPE melanoma samples collected during the oncology care and follow-up. The collected samples were submitted for histopathology and protein analysis by high-resolution mass spectrometry. Proteins and pathways were detected indicating survival and therapy response.

\section{Materials and Methods}

\subsection{Patient Cohort}

The melanoma samples were collected from the Department of Dermatology and Immunology of the University of Szeged. This study was conducted according to the guidelines of the Declarations of Helsinki, and approved by the Hungarian Ministry of Human Resources, Deputy State Secretary for National Chief Medical Officer, Department of Health Administration. The protocol code is MEL-PROTEO-001, the approval number is 4463-6/2018/EÜIG and the date of approval is 12 March 2018. 
In the study, 52 patients with primary and 25 patients with metastatic melanoma were enrolled, 53 primary and 37 metastatic melanoma samples were analyzed, and 8 paired primary-metastasis correlations were compared.

The patients were selected from 2005 to 2020 whose primary melanoma or melanoma metastasis is archived in paraffin-embedded tissue blocks. All primary tumors resulted in loco-regional and/or disseminated disease. The histopathological slides were made from formalin-fixed, paraffin-embedded (FFPE) blocks.

A total of 90 samples were collected with the clinical information, including gender (39 males and 38 females), age at primary tumor (mean $=64.33 \mathrm{yrs}, \mathrm{SD} \pm 10.9, n=77$ (in 3 cases the age at primary was not available), age at metastasis (mean $=65.93 \mathrm{yrs}, \mathrm{SD} \pm 10.93$, $n=77$ ), age at collection date (mean $=64.98$ yrs, $\mathrm{SD} \pm 10.81, n=77$ ), localization of primary tumor (trunk $=17$, lower limbs $=13$, upper limbs $=8$, head and neck region $=14$, acral region $=1$ ) and metastases, long-term follow up data of the patients: disease-free survival (DFS) (mean $=17$ months, $\mathrm{SD} \pm 28, n=77$ ), progression-free survival (PFS) (mean $=42$ months, SD $\pm 39.27, n=77$ ), overall survival (OS) (mean $=51$ months, $\mathrm{SD} \pm 45.3, n=77$ ) (in 3 cases the DFS, PFS and OS were not available). Histological subtypes (SSM-superficial spreading melanoma, NM-nodular melanoma, ALM - acrolentiginous melanoma, LMM-lentigo maligna melanoma etc.), pathological TNM staging (according to the 8th edition American Joint Committee on Cancer (AJCC8) cancer staging system), histological parameters of the primary tumor (Clark level, Breslow level, presence of regression and ulceration), BRAF status (WT, BRAFV600E, BRAFV600K and D5879). Twenty-two patients received immunotherapy (CTL4-inhibitors-ipilimumab, PD-1 inhibitors-pembrolizumab and nivolumab, PD-L1 inhibitor-atezolizumab); 15 patients received targeted therapy (BRAFi (dabrafenib and vemurafenib) and MEKi (trametinib and cobimetinib); 59 patients received other therapies (irradiation, chemotherapy, IFN therapy and electrochemotherapy); and 18 patients did not receive any treatments. Three patients received both immunotherapy and targeted therapy. OS was calculated from the date of diagnosis of primary melanoma to the date of last follow-up (long survival group, live status marked with $1, n=34$ ) or death (short survival group, live status marked with 0, $n=43$ ), PFS was calculated from the date of diagnosis of primary melanoma to the date of progression, DFS was calculated from the date of diagnosis of primary melanoma to the date of first metastasis. The clinicopathologic data of the samples were collected in an Excel file for statistical analysis.

\section{Survival Analysis of Patient Cohort}

Kaplan-Meier (KM) survival analyses were conducted with the disease-free survival (DFS), progression-free survival (PFS) and overall survival (OS) (measured with months) using different statistical approaches (KM including log-rank, Breslow and Tarone-Ware tests). Kaplan-Meier survival analyses and figures showing $p$-values, quartile values, mean values and $95 \%$ confidence intervals were produced by IBM SPSS statistics package (26.0 version) software. Alpha was set to 0.05 and $p$-values less than 0.05 were considered significant.

\subsection{FFPE Sample Preparation and Histology Validation}

A total of 90 primary and metastasis samples were collected retrospectively and obtained by excision or biopsy of melanoma or metastatic organs and archived by formalinfixed and paraffin-embedded (FFPE) routine methodology. Stepwise sectioning of the FFPE tissues was conducted by a conventional microtome. The setting is adjusted to the slice thickness at $10 \mu \mathrm{m}$. FFPE tissue sections were placed on glass slides and stained with hematoxylin and eosin. After staining, the slices were placed in an automated slide scanner system (3D Histech Ltd., Budapest, Hungary). The slides were evaluated for the tumor and stroma ratio.

\section{FFPE Sample Processing}

The FFPE tumor slides were incubated for $10 \mathrm{~min}$ at $97^{\circ} \mathrm{C}, 500 \mathrm{rpm}$ in $1 \mathrm{~mL}$ of EnVision Agilent solution (Agilent, CA, USA) (dilution 1:50). After incubation, the samples 
were centrifuged $\left(14,000 \times g, 3 \mathrm{~min}, 4^{\circ} \mathrm{C}\right)$, discarding the supernatants. For a complete deparaffinization, this step was repeated four times until the supernatant was cleared out. Samples were resuspended in $500 \mu \mathrm{L}$ of protein extraction buffer ( $25 \mathrm{mM} \mathrm{DTT}, 10 \%(w / v)$ SDS in $100 \mathrm{mM}$ TEAB pH 8.0) incubating for $1 \mathrm{~h}$ at $99{ }^{\circ} \mathrm{C}$ in constant agitation (500 rpm). Next, tissues were sonicated (40 cycles, $15 \mathrm{~s}$ on/off) in the Bioruptor (Diagenode, Denville, NJ, USA) followed by centrifugation at $20,000 \times g$ for $20 \mathrm{~min}$ at $18{ }^{\circ} \mathrm{C}$. Supernatants were stored at $-80^{\circ} \mathrm{C}$ until further use, separating a sample aliquot for protein determination (660 nm Protein Assay/Ionic Detergent Compatibility Reagent-Thermo Fisher, Rockford, IL, USA).

\subsection{Protein Digestion}

Before protein digestion, a spike-in of Lysozyme C protein from Gallus (Sigma-Aldrich, St. Louis, Missouri, USA) was done in each sample for batch normalization (100:1 ratio, Sample/Lysozyme C). Protein alkylation was performed with iodoacetamide $50 \mathrm{mM}$ in dark condition for $30 \mathrm{~min}$ at room temperature. S-Trap ${ }^{\text {TM }}$ 96-well plate was used for sample digestion (c). First, an incubation with LysC (enzyme: substrate, 1:50) was added to the samples for $2 \mathrm{~h}$, at $37^{\circ} \mathrm{C}$, followed by trypsin (enzyme: substrate, 1:50) incubation overnight at $37^{\circ} \mathrm{C}$. The samples were acidified with $100 \%$ formic acid (FA) ( $\sim 10 \%$ final concentration) to stop the digestion process. Peptides were dried in a speed-vac (Thermo Fisher Scientific) and resuspended in a solution of $0.1 \%$ trifluoroacetic acid (TFA) $/ 2 \%$ acetonitrile (ACN). The quantitative colorimetric peptide assay (Pierce ${ }^{\mathrm{TM}}$ - Thermo Ficher, Rockford, IL, USA) was used for the determination of peptide concentration. Peptide samples were spike-in with an indexed retention time (iRT) standard (Biognosys, Schlieren, Switzerland) for the control quality of batch normalization.

\subsection{LC-MS/MS Analysis}

Two micrograms of the peptides was injected into the UltiMate 3000 RSLCnano system (Dionex, Sunnyvale, CA, USA) using a trap-column (Acclaim ${ }^{\circledR}$ PepMapTM 100, $75 \mu \mathrm{m} \times 2 \mathrm{~cm}$, nanoViper $2 \mathrm{Pk}$, Thermo scientific) and an analytical column (PepMap RSLC C18, $2 \mu \mathrm{m}, 100 \AA, 75 \mu \mathrm{m} \times 25 \mathrm{~cm}$ Thermo Scientific) online to Q-Exactive HF-X mass spectrometer instrument (Thermo Scientific). Chromatographic separation of peptides was performed in a linear gradient of $160 \mathrm{~min}$ in a flow rate of $300 \mathrm{~nL} \mathrm{~min}{ }^{-1}$, using $0.1 \% \mathrm{FA}$ (buffer A) and $0.1 \%$ FA/80\% ACN (buffer B) solution. The solvent gradient was: 0-3 min $2 \%$ of $\mathrm{B}, 3-115 \min 25 \%$ of $\mathrm{B}, 115-125 \min 32 \% \mathrm{~B}, 125-132 \min 45 \% \mathrm{~B}, 132-14090 \% \mathrm{~B}$ 140-145 90\% B.

The spectra were acquired in a data-dependent acquisition mode. Spray voltage was set at $1.85 \mathrm{kV}$. The full scan resolution was 120,000 , considering a dynamic exclusion time of $30 \mathrm{~s}$. The AGC and injection time (IT) were $3 \times 10^{6}$ and $50 \mathrm{~ms}$, respectively. The 20 most intense ions were selected for fragmentation using a higher-energy collisional dissociation. The MS2 resolution was 15,000, using an AGC of $1 \times 10^{5}, 50 \mathrm{~ms}$ of IT, and normalized collisional energy of $28 \mathrm{EV}$.

\subsection{Database Searching}

Data were searched against the UniProt human database $(2020 / 05 / 26)$ and two spectral libraries such as the Proteome tools HCD 28 PD and NIST Human Orbitrap HCD using the Proteome Discoverer 2.4 software (Thermo Scientific). Two missing cleavages for trypsin digestion were allowed. The precursor and fragment mass tolerance were set to $10 \mathrm{ppm}$ and $0.02 \mathrm{Da}$, respectively. Briefly, the pipeline includes two nodes using the spectrum confidence filter tool. The first node, the dynamics modification at the peptide level, includes the methionine oxidation and lysine methylation of peptides. The acetylation, met-loss, and met-loss + acetyl of the protein N-terminal were also set as dynamic modifications at the protein level. Finally, the carbamidomethylation of cysteine was included as a static modification. The second search node considers the methionine oxidation and lysine methylation of peptides and the protein $\mathrm{N}$-terminal acetylation as 
dynamic modifications. Static modifications such as cysteine carbamidomethylation were also considered. In addition, the Minora Feature Detector and the Feature Mapper nodes were used as a tool for the data search workflow.

\subsection{Data Normalization and Batch Effect Correction}

No imputation was performed on either a peptide or a protein level during data processing. The raw protein intensities were $\log _{2}$-transformed and median-normalized (by centering around the global median, which included all non-zero values of the whole dataset). The spike-in protein Lysozyme $C$, which was added in equal amounts during sample preparation and therefore expected to have constant expression across the samples, exhibited a continuous drift in its intensity according to the injection order, and this batch effect was visible also on the PCA plot of the samples (Figure S1A,D). To correct for this technical factor, a continuous batch correction method implemented in the proBatch $\mathrm{R}$ package (v. 1.6.0) [14] was utilized. Firstly, discrete batches were defined based on the injection order: 1-24, 25-49, 50-74, 75-90, and then a non-linear trend was fitted to the Lysozyme $C$ expression values in each batch (Figure S1B). The locally estimated scatterplot smoothing (LOESS) span was set to 0.9, leading to a smoother fitted curve, which is less prone to overfitting. This trend, estimated based on the spike-in protein, was then subsequently subtracted from all protein intensity values. The efficiency of the batch correction was checked on the iRT peptides' intensity, which was added to the samples before the MS measurement in equal amounts. The iRT peptide levels were found to be very similar after batch correction in the samples; moreover, the PCA plot of the samples now showed no separation based on injection order (Figure S1C,D). Moreover, a separation between tumor samples retrieved from cutaneous (Cut) and lymph node (LN) origins arose after batch correction (Figure S1D), showing sample grouping according to tissue type. Therefore, the normalized and batch-corrected expression values were used for subsequent analyses. Intensity distributions of the samples before and after median-normalization and after batch effect correction are visualized in Figure S1E.

All data post-processing steps and subsequent statistical tests were performed in $\mathrm{R} \mathrm{v}$. 4.0.4 using RStudio v. 1.4.1106. Visualizations were made using ggplot2 v.3.3.3 [15], ggbiplot v.0.55 [16], cowplot v.1.1.1 [17], gridExtra v.2.3 [18] and ComplexHeatmap v.2.6.2 [19].

\subsection{Proteomics Data Analysis}

Unsupervised clustering of the samples was performed using proteins that have intensity values in at least $50 \%$ of the samples. The hierarchical clustering was done on the Z-score normalized protein expression table using Euclidean distance and complete linkage. The Euclidean distance was selected as it gives equal weight to all proteins, and the best results were achieved using complete linkage. Detection of individual clusters was done via the dynamic tree cutting method, a method implemented in the dynamicTreeCut $\mathrm{R}$ package (v. 1.63-1), with the following settings: $\operatorname{minClusterSize}=0$, method $=$ hybrid, deepSplit $=4$. The dynamic tree cutting [20] over constant-height tree cutting was selected as it can identify nested clusters (see Figure S2A), and it automatically detects the optimal number of clusters. Overrepresentation of any clinical or histopathological characteristics in the sample clusters was determined using one-sided Fisher's exact test. Alpha was set to 0.05 and nominal $p$-values less than 0.05 were considered significant, but clinical/histopathologic traits with nominal $p$-values between 0.05 and 0.01 were also discussed.

For the proteomic characterization of the sample clusters, a one-way Analysis of Variance (ANOVA) test with pairwise Tukey's honest significant difference (HSD) posthoc tests were performed to identify proteins showing differential expression between the sample clusters. No prior filter for valid values was applied on the expression table, but statistics was performed only on proteins that were quantified in at least $80 \%$ of the minimum 2 sample clusters. Benjamini-Hochberg adjusted $p$-values values (FDR) of ANOVA tests less than 0.05 were considered significant. Unsupervised hierarchical clustering of the top 1000 most differentially expressed proteins was performed using 
Euclidean distance and complete linkage, followed by dynamic tree cutting with the settings $\operatorname{minClustersize}=50$, method $=$ hybrid, deepSplit $=0$. Pathway enrichment analysis of the resulting protein clusters was conducted using Kyoto Encyclopedia of Genes and Genomes (KEGG) [21] and Reactome [22] databases.

Proteins showing differential expression between primary and metastasis samples were examined via $t$-tests (independent $t$-tests for the whole cohort and paired $t$-tests for the paired primary and metastasis samples). Only proteins with min. $80 \%$ valid values in both groups were included in the analysis. For the paired $t$-test, the $\log 2$ fold changes (FCs) were calculated by calculating log2FC between the paired samples, and then taking the average of those paired differences. Proteins were ranked based on the log2FC $\times-\log 10$ nominal $p$-value for the subsequent pre-ranked gene set enrichment analysis (pGSEA). Linear relationship between stage and protein expression was assessed using Analysis of Covariance (ANCOVA), where both stage as a numerical variable (2, 3 and 4 ) and tumor type as a categorical variable (primary or metastasis) were included in the model. Only proteins with min. $80 \%$ valid values in all three stages were considered in the analysis. Regression coefficients for stage and nominal $p$-values were extracted, which were used to rank the proteins (coefficient $\times-\log 10$ nominal $p$-value) for the pGSEA. The pGSEA was performed via the clusterProfiler R package [23]. The GO biological process, KEGG, Reactome and Wikipathways gene sets were utilized for the analysis. The gene sets were downloaded from the Molecular Signatures Database v7.4 [24-26]. For primary vs. metastasis comparisons, the FDR-corrected pGSEA $p$-value $<0.05$ was considered significant, whereas for stage comparisons, nominal $p$-value $<0.05$.

Proteins that are potential predictors of long or short progression-free survival after the patients received immunotherapy or targeted therapy were detected via survival analysis. For this, multiple Cox regression models were fitted to the progression-free survival data in both the immunotherapy and targeted therapy subgroup. Individual protein expression data were used as a covariate in the model (provided that the protein had $\min .80 \%$ valid values within the therapy subgroup's samples), and samples were stratified based on the tumor type (Primary or Metastasis). Alpha was set to 0.05 and nominal $p$-values less than 0.05 were regarded as significant. Proteins where the proportional hazards assumption was not met (checked by the cox.zph function of the survival $\mathrm{R}$ package, $p$-value $<0.05)$ were deleted from the list of significant proteins. Protein networks were drawn via STRING (v.11.5) [27]. Overrepresentation analysis of GO biological processes [28] and KEGG pathways, as well as interaction network construction (confidence cutoff 0.4 ) using the significant proteins, was performed in Cytoscape (v.3.8.2) [29]. The processes showing FDR $<0.05$ were visualized and used for biological interpretation.

\subsection{Data Availability}

The mass spectrometry proteomics data have been deposited to the ProteomeXchange Consortium via the PRIDE [1] partner repository with the dataset identifier PXD028930. Project name is Proteomic Analysis on Paraffine Archived Melanoma. Project accession is PXD028930, project DOI is not applicable, username is reviewer_pxd028930@ebi.ac.uk, and password is TaHGkBGm.

Proteomic data summary, ANOVA and Tukey's HSD test results of sample cluster comparisons, Cox regression analysis results for immunotherapy and targeted therapy subgroups, $t$-test results (paired/independent) for primary vs. metastasis comparisons as well as ANCOVA results for the comparison of disease stages are provided in Supplementary Document S1. The scripts used for the proteomic data normalization, batch effect correction and statistics are available at https://github.com/bszeitz/MM_pilot (accessed on 21 November 2021). Clinical information of individual patients cannot be provided due to the ethics restrictions.

\subsection{Figure Illustration}

Some of the figure illustrations were created with BioRender 2021 software. 


\section{Results and Discussion}

\subsection{The Clinical Significance of the Study}

Clinicopathologic Characteristics of the Patient Cohort

The applied retrospective cohort is composed of 90 melanoma samples from 77 patients with mainly progressed melanoma. The samples consist of 53 primary and 37 metastatic melanoma samples representing both lymphatic and cutaneous metastases and eight pairs of primary-metastasis correlation. The clinicopathologic data of the samples are summarized in Table 1. From the investigated cases, $51 \%$ were male individuals and $56 \%$ of the participants were not alive at the collection date (short survival group). The melanomas were stratified according to the 8th edition of the American Joint Committee on Cancer (AJCC8) staging system [30] and the 3rd edition of the WHO classification [31]. The metastatic cohort had a predominance of patients who had stage III $(40 \%)$ at the time of primary tumor sampling (Table 1). Furthermore, data of the BRAF mutation were also collected from the primary and metastatic samples, with 51\% coming from the mutated BRAFV600 state.

The 90 analyzed melanoma samples included $58.9 \%$ primary tumors, $26.66 \%$ locoregional lymph node metastases and $14.44 \%$ cutaneous metastasis samples. The majority of the samples were surgically removed from the trunk $(32 \%)$ and the dominant histotype of the primary tumors were nodular melanoma $(51 \%)$. The tumors spread into the deeper layers of the dermis or subcutaneous layer of the skin in $51 \%$ of the primary samples (Breslow thickness $>4 \mathrm{~mm}$ ) (Table 1).

The examined FFPE tumor samples varied from medium-thick (1-2 mm-pT2) to thick (high risk: $2-4 \mathrm{~mm}-\mathrm{pT}$, very high risk: $>4 \mathrm{~mm}-\mathrm{pT} 4$ ) melanomas with a dominance of the thick/very high-risk cases up to even $22 \mathrm{~mm}$ of thickness. The primary melanomas mainly showed a poorly differentiated solid area reaching at least Clark III level, indicating a vertical growth pattern even at superficial spreading or lentigo melanoma cases. The peritumoral niche varied from lymphocyte- and macrophage-rich areas to regressive, fibrotic, pauci-cell microenvironment. Focal necrotic areas as well as intratumoral pigmentation were assessed. In the lymphatic and cutaneous metastases, variable degrees of alteration in necrotic areas, viable tumor counterparts, tumor burden, and regressive signs were observed. In addition, the tumor/stroma ratios were evaluated in each sample. (Figure 2).

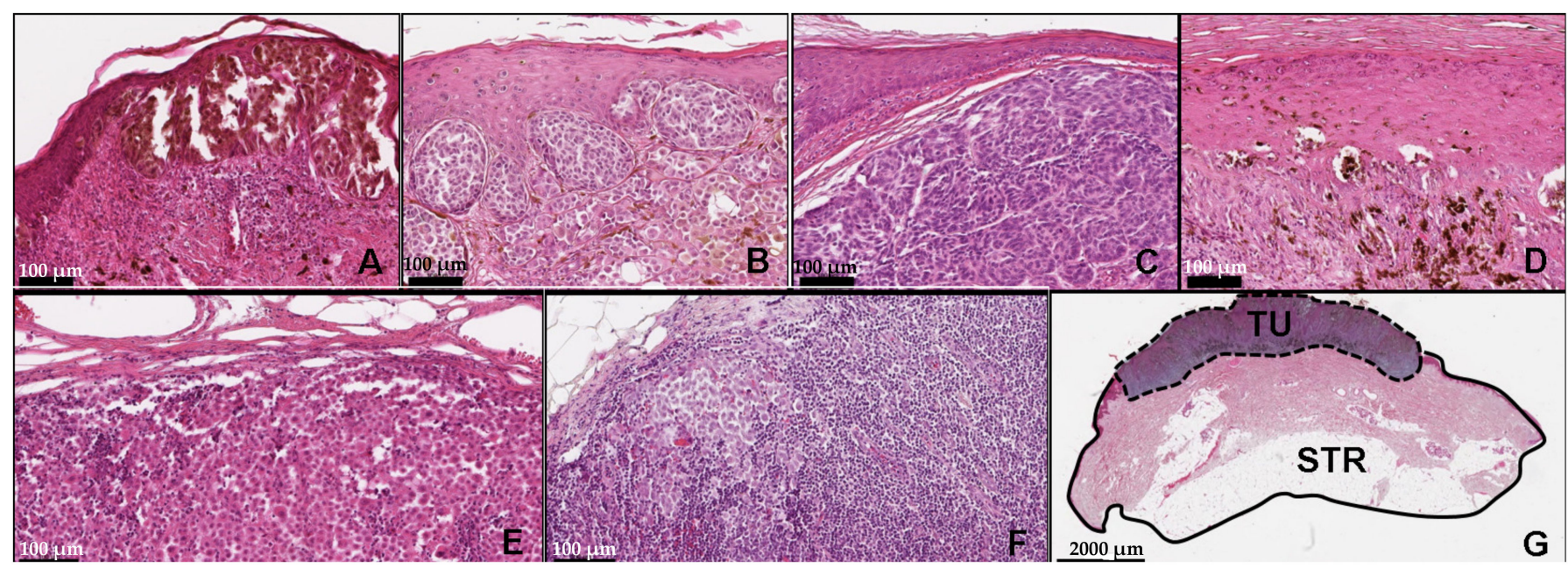

Figure 2. Histopathologic features of the representative cases in the study. Image captures, representing the spectrum of melanoma sample subtypes, including SSM (superficial spreading melanoma) (A), SSM with a vertical involvement (superficial spreading melanoma with vertical involvement) (B), NM (nodular melanoma) (C), ALM (acrolentiginous melanoma) (D), as well as both cutaneous (E) and lymphatic (F) metastases. Each sample also underwent a tumor (TU)/stroma (STR) ratio assessment (G) (HE (hematoxylin-eosin); OM (optimal magnitude) 112×). 
Table 1. Clinicopathologic data of the patient cohort.

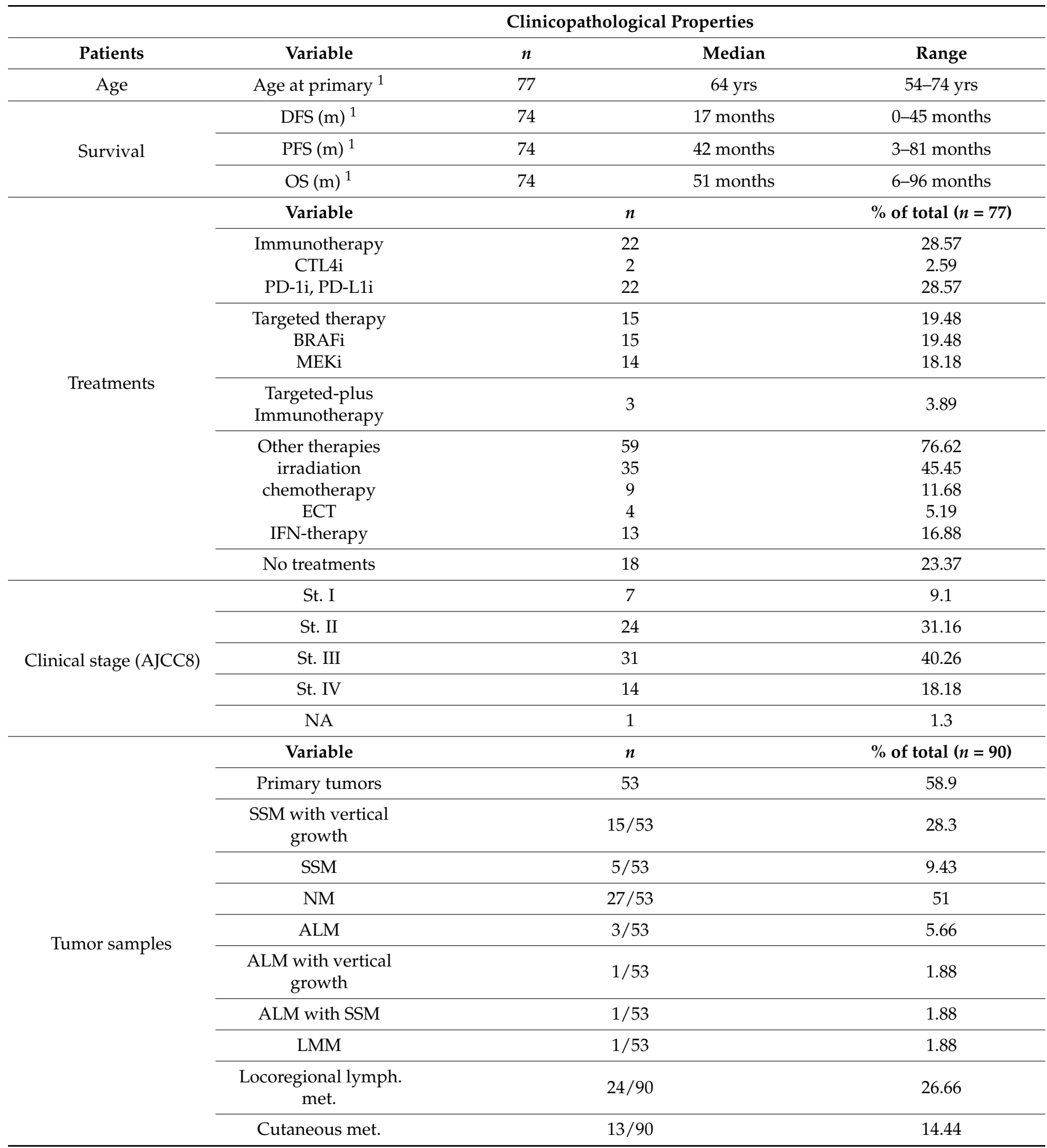

The table represents the clinicopathologic parameters of the patients and their selected primary melanomas and metastases included in the pilot patient cohort. DFS—disease-free survival, PFS—progression-free survival, OS-overall survival; CTL4i-cytotoxic T-lymphocyte associated protein inhibitor, PD-1i-programmed cell death protein 1 inhibitor, PD-L1i-programmed death ligand 1 inhibitor, BRAFi-BRAF kinase inhibitor, MEKi-MEK kinase inhibitor, ECT-electrochemotherapy; AJCC8-The 8th edition American Joint Committee on Cancer staging system; yrs—years; SSM—-superficial spreading melanoma, LMM—lentigo maligna melanoma, NM—nodular melanoma, ALM-acrolentiginous melanoma, NA-no available data. ${ }^{1}$ In three cases, the age at primary diagnosis, the disease-free survival, the progression-free survival and the overall survival were not available. 
3.2. Proteomic Analysis Reveals Sample Clustering According to Relevant Histopathological and Clinical Parameters Associated with Survival and Tumor Progression

A total of 90 samples from 77 patients were analyzed based on their clinical- and global proteomic expression data. Mass spectrometry analysis quantified 7881 protein groups across all datasets (Supplementary Document S1). The unsupervised hierarchical cluster analysis revealed six major sample clusters and one outlier sample labeled as Cluster 0 (Figure 3A). A total of 4437 protein groups showing variability among these clusters were detected (Figure 3A). Enrichment analyses revealed a correlation of one of each cluster to relevant clinical parameters. Cluster 1 corresponded to better overall survival in the interval of 101 to 205 months (Fisher test $p<0.05$ ), Cluster 2 corresponded to samples with the host tissue of cutaneous origin, exclusively (Fisher test $p<0.001$ ), and a tendency for poorer disease-free survival ranging from 11 to 30 months (Fisher test $p=0.0835$ ) was observed. Cluster 3 showed a borderline significant (Fisher test $p=0.0913$ ) enrichment of better progression-free survival values (between 61 and 100 months). On the other hand, samples in Cluster 4 had the host tissue of lymphoid origin (Fisher test $p<0.001$ ), as well as enrichment of the AJCC8 St. IIID stage (Fisher test $p<0.05$ ) and the Breslow level between 4.1 and $8 \mathrm{~mm}$ (Fisher test $p=0.0616$ ) was observed. Moreover, the dominant gender was male (Fisher test $p=0.0703$ ), and a borderline significant association with poor disease-free survival with less than 10 months (Fisher test $p=0.0778$ ) was also noted. In Cluster 5, three out of five samples, all primary tumors, can be categorized into the ALM subtype (Fisher test $p<0.05)$. Moreover, the gender was exclusively female (Fisher test $p<0.05$ ). The AJCC8 St. IIA (Fisher test $p=0.0647$ ), progression-free survival between 11 and 30 months (Fisher test $p=0.0662$ ), and overall survival between 11 and 30 months (Fisher test $p=0.0662$ ) were also borderline enriched. Cluster 6 corresponded to a better disease-free survival ranging from 31 to 60 months (Fisher test $p<0.05$ ); however, these samples exhibited low overall protein coverage, and can therefore be regarded as outlier samples (Table S1). Similarly, Cluster 0 was built up from one outlier sample; therefore, associations with clinical and histopathological characteristics were not evaluated (Figure 3A).

To unveil the molecular signature behind the sample clusters, we performed a biological pathway analysis considering the top 1000 most variable proteins (ANOVA FDR $\left.<1.05 \times 10^{-4}\right)$. Figure $3 \mathrm{~A}$ shows the protein clusters obtained by hierarchical clustering, and Figure $3 \mathrm{~B}$ shows the main biological functions associated with the sample clusters. One of the main findings was the downregulation of protein cluster 2 in sample cluster 4 and their upregulation in sample clusters 5 and 6 . The closer data analysis revealed that most of the proteins are related to the cellular matrix function, such as desmoglein-1, desmocolllin-2, and dystonin, specifically in the desmosome and the hemidesmosome assembly. The decrease in proteins related to desmosome are linked to tumor progression in several types of cancers [32,33].

Furthermore, for example, desmoglein-1 was proposed as a new therapy candidate due to its participation in the invadopodia formation, promoting tumor invasion and metastasis development [34]. Divergent results have been found concerning the desmocolllin-2 role in metastasis. Evidence suggests that the reduction in the expression of this protein promotes cellular migration and tumor invasion [35-37], while other studies affirm that the upregulation of desmoglein 2 promotes vasculogenic mimicry and metastatic spread in melanoma [38]. On the other hand, the decline of proteins associated with the hemidesmosome components was also related to metastasis progression and poor clinical outcomes [39], and it correlates with our results since we have seen the downregulation of these proteins in sample cluster 4 with poor disease-free survival.

Protein clusters 1, 4, 5, 6, 7 and 10 stand out by the dramatic decrease in the sample cluster 6 , the better prognosis group. Alterations in mitochondrial translation and RNA metabolism are the most enriched pathways. Detailed examination of the biological significance of these protein changes displays the alteration of the mitochondrial ribosomal complex (e.g., MRP-S7, MRP-L12, MRP-L15) and RNA regulation (e.g., integrator complex). Furthermore, mitoribosomal proteins (MRP) are reported as key drivers in regulating 
apoptotic signaling beyond their classical protein translation role [40]. Interestingly, the abnormal protein expression of MRP has been described in several tumors, and currently, they are proposed as novel targets for disease diagnosis and treatment [41]. In the same way, the alteration of the MRP-S7 gene expression is strongly correlated to the metastatic process in the osteosarcoma [42], while MRP-L12 was proposed as a potential predictive biomarker for glioblastoma [43]. In addition, MRP-S15 was suggested as one of the predictor markers of tumor recurrence and treatment failure in breast cancer patients [44]. In parallel, the detriment in the abundance of the important components of transcription machinery previously linked to cancer malignancy, such as the subunits of the integrator complex [45].
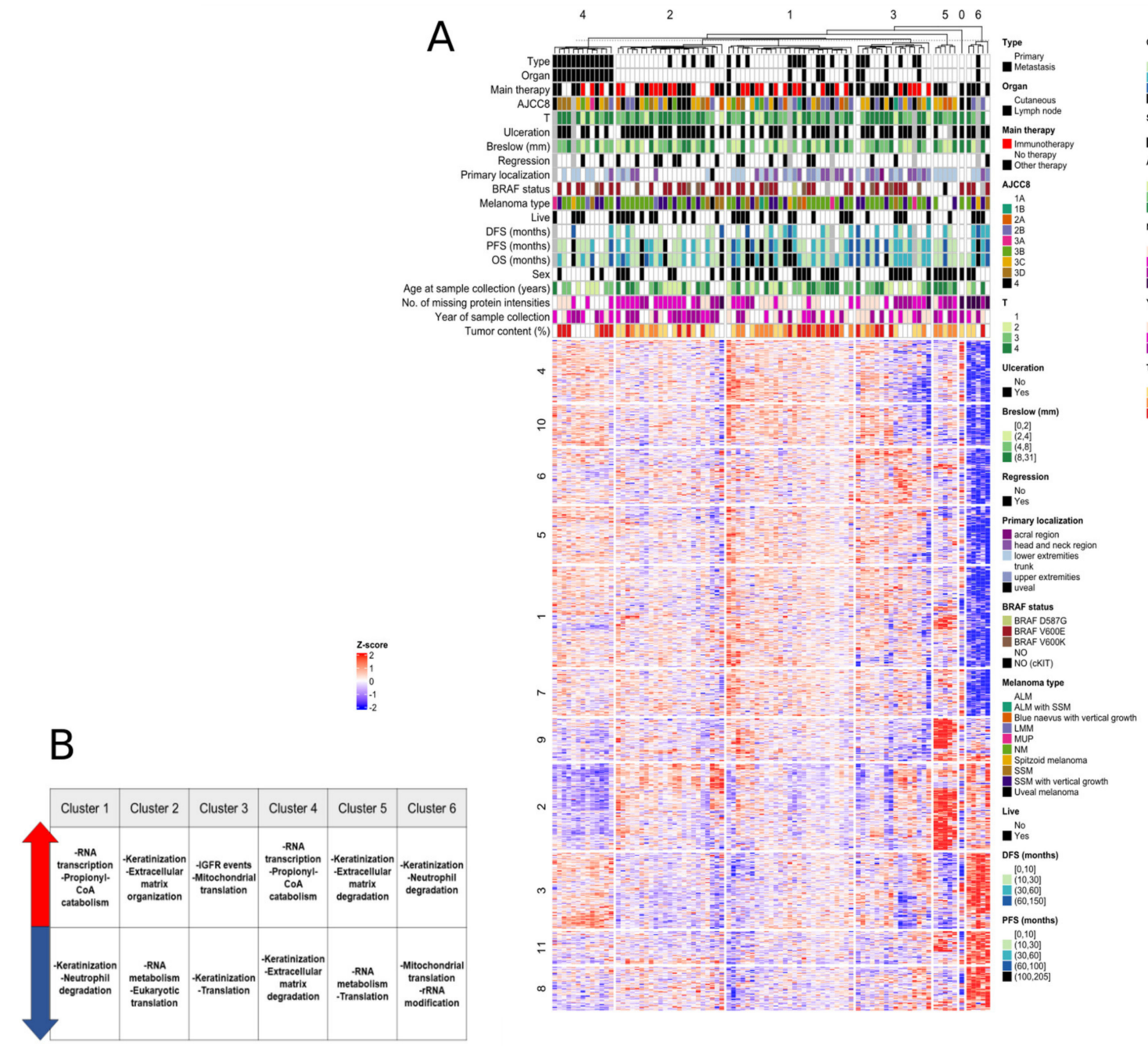

Figure 3. The protein expression patterns in the sample clusters. (A) The heatmap of the six detected sample clusters with the clinicopathologic data and the protein expression pattern (top 1000 most variable proteins, ANOVA FDR $<1.05 \times 10^{-4}$ ). (B) The main biological functions linked to the sample clusters. DFS-disease free survival, PFS-progression-free survival and OS-overall survival. Clinical stage based on AJCC8. SSM—superficial spreading melanoma, LMM-lentigo maligna melanoma, NM—nodular melanoma, ALM—acrolentiginous melanoma.

On the contrary, protein clusters 3, 8, and 11 are highly increased in sample cluster 6. These protein groups are mainly involved in the RNA metabolism in response to DNA damage, especially in the increase in proteins related to TP53/P53 response (e.g., probable ATP-dependent RNA helicase DDX5, heterogeneous nuclear ribonucleoprotein K, 40S ribosomal protein S3). For example, it was demonstrated that 40 S ribosomal protein S3 protects p53 against MDM2 ubiquitination and proteasome degradation [46]. In addition, we noticed the increment of proteins linked to phagocytosis and membrane remodeling, 
such as merlin, reported as a tumor suppressor reducing the migration and proliferation of metastatic melanoma [47]; this finding is in agreement with the clinical characteristics of sample cluster 6 with better disease-free survival.

Similarly, we also discovered an increase in proteins within the complement system, and coagulation cascade (e.g., complement C3, fibrinogen), which are part of the tumor microenvironment, playing crucial functions in promoting tumorigenesis and a scaffold for the binding of tumor cell growth [48-50].

\subsection{Imbalance of Immune-Related Proteins as Molecular Fingerprint of the Sample Clusters with Varying Survival Outcomes}

The bioinformatics analysis of the proteomic profiles captures two different sample clusters with distinct overall survival (i.e., cluster 1 and cluster 5$)$. The comparison between cluster 1 vs. cluster 5 detected the downregulation of 316 protein groups (Figure 4A), mainly linked to the immune system (e.g., CD33, ARGI1, NKAP). In this case, the imbalances of these immune-related proteins were associated with poor clinical outcomes. For instance, the alteration in the abundance of proteins such as CD33, a transmembrane receptor expressed by myeloid lineage, was correlated with poor therapy responsiveness and disease progression [51,52]. On the other hand, the increase in ARGI1 in the tumors usually correlates with metastases and worse clinical prognosis [53]. Moreover, the expression of NKAP is negatively correlated with the levels of Notch target genes, which are essential for T-cell development [54]. Moreover, the NKAP acts as an oncogene and is connected with a poor outcome in cancer patients. It was demonstrated that NKAP knockdown suppresses the proliferation of HCT116 and HT-29 cells, inducing apoptosis $[55,56]$. On the other hand, 74 protein groups were found upregulated. Similarly, we detected the increase in proteins related to the immune system with anti-tumorigenic effects, such as GTPase IMAP family member 5 (GIMA5), described as a promoter of CD4+ T-cell survival and homeostasis [57,58]. GIMA5 also suppresses the proliferation of lung cancer cell lines [59]. In addition, we observed the increment of proteins such as mitogen-activated protein kinase 2 (MAP4K2), usually expressed in B cells of the germinal center and macrophages, which activates the c-Jun N-terminal kinases [60]. Similarly, we detected the different expression of PIK3CA between the sample clusters. This protein participates in several signal transduction pathways, such as the regulation of the T-cells, and cell signaling, but especially those that impact cell proliferation, survival, and apoptosis in tumors [61,62].

\subsection{Protein Translation and Immune System Stand out in the Proteome Profile of the Sample Clusters with Different Progression-Free Survival}

The comparison of sample clusters (Figure 4B) with different progression-free survival (cluster 3 vs. cluster 5) shows a decrease within 333 protein groups. The translation pathway was found highly enriched, together with the differential expression of several immune-related proteins. As mentioned above, some of the ribosomal proteins that we identified as differentially expressed between sample clusters in this study also have an alternative function to protein translation, such as MRP-L12 (previously described) as well as RPS12 that triggers metastasis development through the activation of the Akt/mTOR/cMyc signaling pathway in cervical cancer cell lines [63]. Moreover, we can also highlight the decrease in key immune-regulating proteins, such as l-amino-acid oxidase (OXLA), a critical immune regulator inhibiting the proliferation of the peripheral blood mononuclear cells and CD8+ T-cells. The immune response is thereby suppressed in the tumor microenvironment, contributing to tumor cell invasion [64]. In parallel, we also detected the increase in 108 protein groups, mainly related to the growth signaling cascade such as the SHC-transforming protein 1, previously reported as an important factor in the metastatic progression of breast cancer [65]. Moreover, we detected proteins such as E3 ubiquitin-protein ligase (RNF114) and mitogen-activated protein kinase kinase kinase 7 (MAP3K7) involved in mechanisms related to tumor necrosis factor receptor-associated factor 6 (TRAF6), a well-established protein that plays a critical role in melanoma metasta- 
sis [66]. RNF114 regulates the ubiquitination of TRAF6 [67], while TRAF6 mediates the signal transduction of MAP3K7 in the activation of the nuclear factor-kappa B [68].

A)

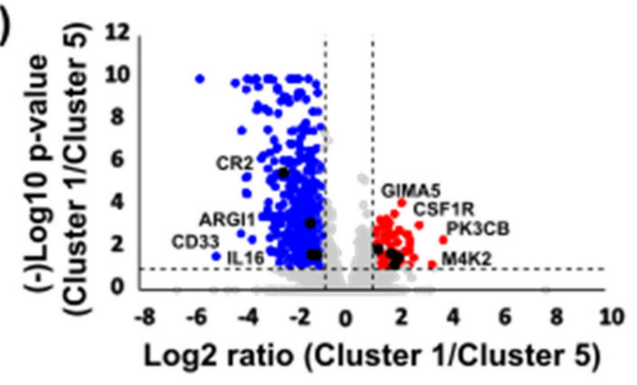

C)
ARMC10

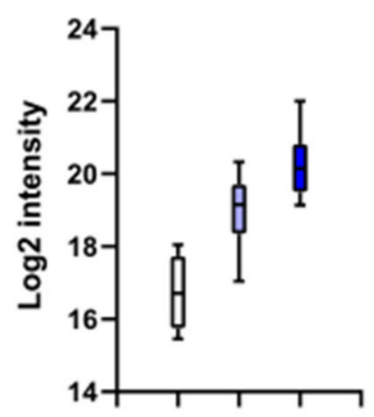

ITGA5

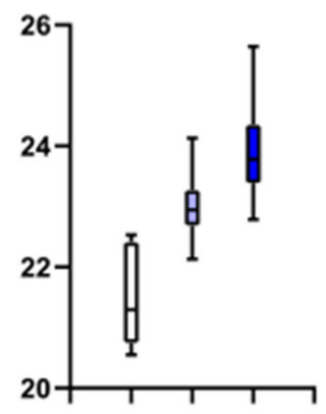

CAMK4
B)

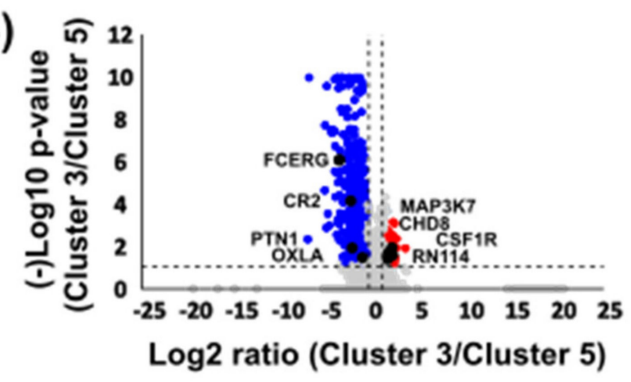

CDK2

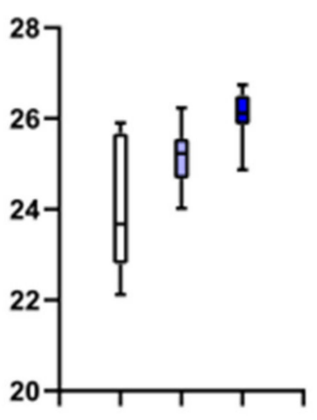

WIPI1

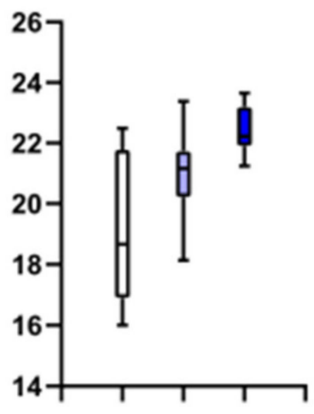

AlF1

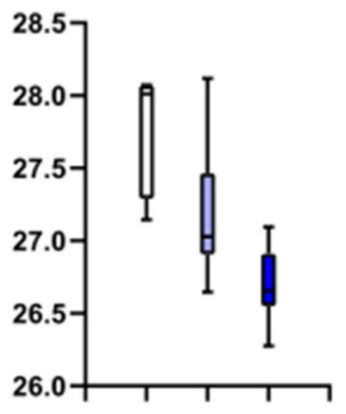

PPIF
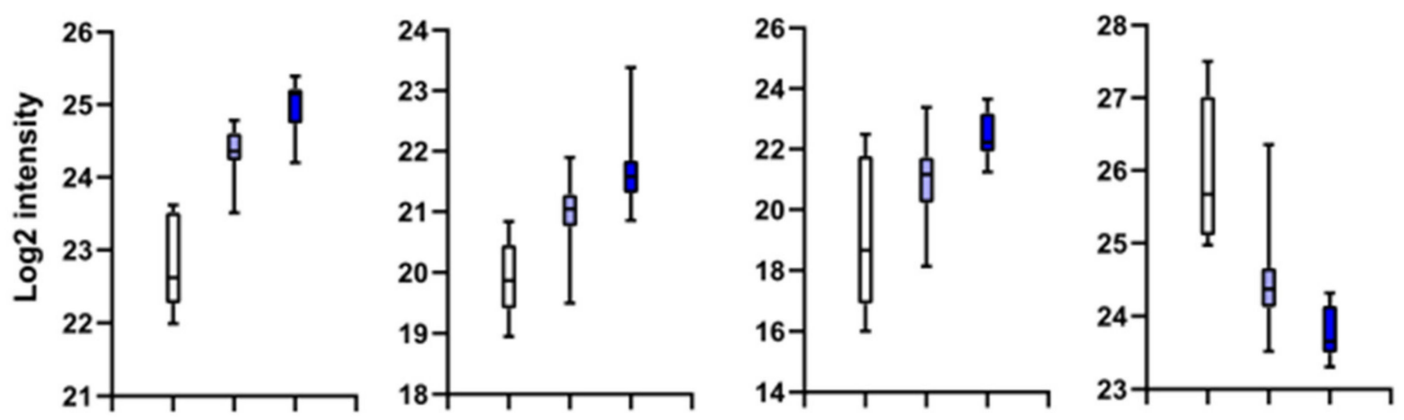

\section{$\square$ Cluster $6 \quad \square$ Cluster $2 \square$ Cluster 4}

Figure 4. The main up- and downregulated proteins in the sample clusters. (A) Volcano plot of sample cluster comparison (between sample cluster 5 and sample cluster 1) with diverse overall survival. (B) Volcano plot of sample cluster comparison (between sample cluster 5 and sample cluster 3) with different progression-free survival. (C) Examples of the differential expression of the proteins between sample cluster 6, 4 and 2 with different disease-free survival.

\subsection{Proteome Mapping Detects Gradual Proteomic Changes in Sample Clusters Exhibiting Different Disease-Free Survival}

To evaluate the proteins that could potentially impact the disease-free survival of our sample cohort, we contrasted the protein changes across three sample clusters with good (Cluster 6) median (Cluster 2) and inferior (Cluster 4) clinical endpoints. Despite sample 
heterogeneity, data analysis shows a significant alteration of 90 protein groups, of which 49 and 7 proteins tend to increase and decrease across these sample clusters, respectively.

Figure $4 \mathrm{C}$ displays an example of the main differential expressed proteins between the sample clusters in our analysis. Proteins with a tendency to increase are mainly related to RNA processing, such as ribosomal proteins. Similarly, we also detected a key marker such as TRAF6, as well as relevant proteins for the cell cycle control and apoptosis such as cyclin-dependent kinase 2 (CDK2), crucial for the proliferation of melanoma cells [69,70], and armadillo repeat-containing protein 10 (ARMC10) linked to cell proliferation and the inhibition of p53 [71,72]. A gradual decrease in apoptosis-inducing factor 1 (AIF1), a wellknown proapoptotic factor, was found to be aligned with early metastasis [73], in addition to peptidyl-prolyl cis-trans isomerase $\mathrm{F}$, an essential component of the mitochondrial permeability transition pore [74].

\subsection{Proteomic Differences with Regard to Tumor Type and Clinical Stage}

In the metastatic cohort, the primary melanoma and their corresponding metastasis samples from eight patients (paired dataset) were compared based on the differences of their proteomic expression profile (via paired $t$-tests); additionally, differences in primaries and metastases from the whole cohort (full dataset) were examined by independent $t$-tests. Pre-ranked Gene Set Enrichment Analysis (pGSEA) outlined a strong agreement between the two results (Figure S2B), indicating that primary vs metastasis differences found in paired samples is globally true for the primary vs metastasis differences in the full dataset as well. The B-cell receptor signaling pathway, endoplasmic reticulum calcium ion homeostasis pathway and the chromatin organization pathway were significantly activated (pGSEA $p$-value $<0.05$ ) in metastatic tumors according to both the paired and full dataset, whereas keratinization pathways, epithelial and epidermal cell differentiation pathways, the epidermis development pathway, collagen fibril organization, the extracellular matrix organization pathway, the myeloid leukocyte mediated immunity pathway and the neutrophil degranulation pathway were strongly activated (pGSEA $p$-value $<0.05$ ) in primary tumors.

In connection with the clinical stages, we have also identified proteins showing up-and downregulation from AJCC8 stage II to stage IV while accounting for tumor type (primary/metastasis differences). A total of 147 proteins were significantly upregulated, and 152 proteins were downregulated in the higher clinical stage (Figure S2C). Pathway analysis through pGSEA showed a concordant upregulation of proteins in the formation of humoral immune response, the regulation of immune effector process, the regulation of humoral immune response, and the regulation of complement activation such as CD46, TRAF6 as well as MCM9 protein in DNA repair mechanisms. The downregulation of the PELO protein in epithelial cell differentiation and the PSMD3 protein in the negative regulation of the canonical WNT signaling pathway (Figure S2C or 2D) was also detected. It is known that CD46 contributes to complement activation and humoral immune response [75], and the overexpression of this protein correlates to the suppression of IFN $\gamma$ and TNF $\alpha$ production on $\gamma \delta$ T-cells and it can further contribute to the formation of a pro-tumor environment, indicating progression through clinical stages. [76-79] The elevated expression of TRAF6 protein during the progressed melanoma course is parallel with our previous results where this protein was upregulated in sample clusters with enrichment for short survival. The MCM9 is involved in the DNA repair mechanisms, and it was recently shown that MCM9 protein is highly expressed in metastatic melanomas compared to primary melanomas in the TCGA cohort (TCGA [80]) and is also related to the T stage (histopathologic stage) of the tumor [81].

A downregulated tendency of the PELO protein in the epithelial cell differentiation pathway was seen. This protein has a role in cell cycle control and an unfavorable prognostic marker in breast cancer [82]. The downregulation of PSMD3 protein in the negative regulation of the canonical WNT signaling pathway was also seen from the mild to the severe stages. Interestingly, in other studies, the downregulation of PSMD3 was associated 
with longer survival in glioblastoma multiforme [83] and in melanoma metastasis [84], which is the opposite tendency to what we have seen in our results in the clinical stages.

\subsection{Histopathologic Relations to the Clustered Sample Cohort}

Although the examined high/very-high-risk metastatic setting is generally supposed to indicate a worse prognosis, the proteomic assays could differentiate into clusters based on survival and therapy-response variables among these mainly aggressive cases. Interestingly, neither of the standardized histopathologic parameters (pT, regression, ulceration, etc.) has proven significant differences among the clustered groups, which indicates an independent prognostic value of the deep proteomic assay, as shown in Figure 5.
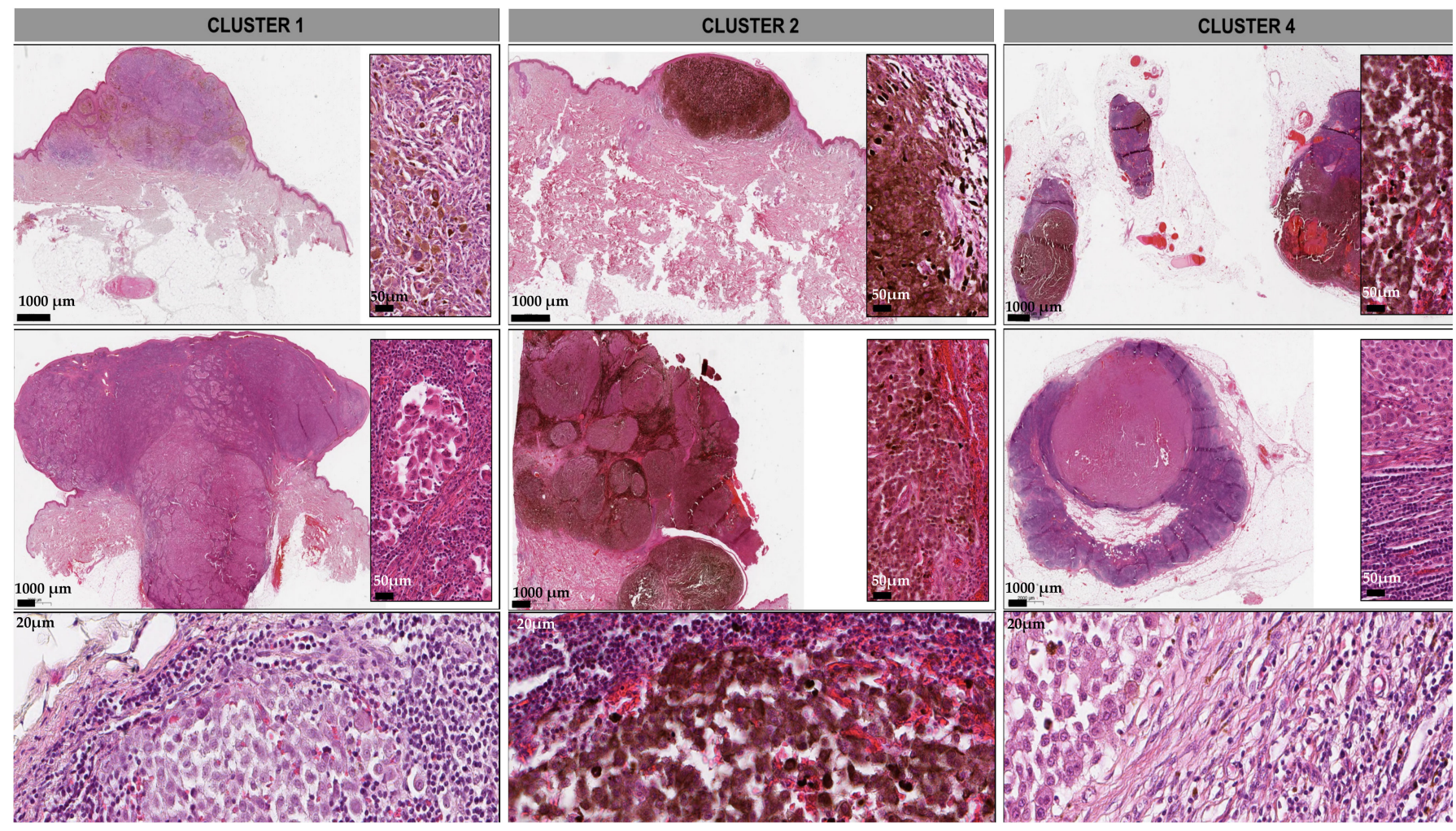

Figure 5. Histopathologic characteristics of the samples in sample cluster 1, 2 and 4 . The image represents the spectrum of the morphology of the examined samples, as Cluster 1 involves vertical growth or de novo nodular melanomas displaying an anaplastic, fusiform or epithelioid phenotype (inserts). Additionally, a few metastases were also included exhibiting the same dedifferentiated morphology. In Cluster 2, similar phenotypes were noted, with a pronounced intratumoral melanin pigmentation. Cluster 4 entailed the majority of the lymph node metastases. OM (optimal magnitude) $112 \times$; HE (hematoxylin-eosin) staining; inserts indicate magnified areas with scale bar $50 \mu \mathrm{m}$ (Table S2).

\subsection{Proteins and Pathways Predicting Therapy Response}

3.8.1. Proteins and Pathways Indicating Worse Outcome in the Immunotherapy-Treated Subgroup

Subgroups of patients were selected based on their primary treatment (immunotherapy, targeted therapy). Twenty-four samples from 22 patients were involved in the immunotherapy subgroup and 20 samples from 15 patients were used in the targeted therapy subgroup. The samples involved in the pilot study were naive to any treatment arms, whilst we are able to observe the protein expression profile prior to any given therapy. Aiming to identify proteins that are possible predictors for the length of progression-free survival (i.e., worse or better response), multiple Cox regression models were established for the progression-free survival, using the protein expression data. The model was stratified based on sample type, i.e., primary or metastasis. The analysis resulted in 401 proteins in 
the immunotherapy subgroup and 260 proteins within the target therapy subgroup, which were found to significantly correlate with survival (Multiple Cox regression $p$-value $<0.05$ ), respectively. The significant proteins are visualized within the heatmap, as shown in Figure 6A,B.

The functional analysis of the proteins (Gene Ontology terms, KEGG pathways, Reactome, Wikipathways) (Figure S4) revealed distinct activated pathways for patients with short/long PFS. For the subgroup with shorter PFS after immunotherapy, the significantly upregulated proteins were related to cellular and metabolic processes (Figure S4), also including the VEGFA-VEGFR2 pathway (Figure 6 (2D)) (KEGG pathway database: FDR < 0.05). The VEGFA and its corresponding pathways activate the endothelial cell growth and vasculogenesis that contributes to the metastatic potential of the tumor [1]. The presence of the VEGFA also suggests a prognostic signature for locoregional lymphatic metastasis [1] In a study, the PD-1/PD-L1 and VEGFA/VEGFC expressions were compared in lymph node metastases to investigate their respective expressions. The study revealed that despite PD-1 expression being correlated with a higher survival rate, VEGFA was connected to the worse prognosis. However, the immunotherapy linkage was not investigated in this case [85]. Our data indicate that an activation of the VEGFA-VEGFR2 pathway can not only be associated with the aggressive behavior of the tumor, but also with the lack of efficient response to immunotherapy. Currently, there are VEGFA blockers used in anticancer therapies. In lung cancer, the application of the anti-angiogenic drugs is a potential choice [86]. The humanized anti-VEGF monoclonal antibody (bevacizumab, Avastin) is the first-line treatment in metastatic colorectal cancer and has already been approved by the FDA [87]. These results raise questions about the role of the VEGFA blockers in the case of melanoma.

In connection with the VEGFA-VEGFR2 pathway, the nitric oxide synthase 3 (NOS3) was also worth noting, since the NO production of this enzyme promotes the VEGF pathway induced angiogenesis [88], tumor proliferation and progression [89]. In line with the previous findings, the downregulation of NOS3 expression (Cox regression test $p<$ 0.05 ) was found in patients with longer progression-free survival with immunotherapy treatment (Figure 6 (2D)).

A similar tendency could be seen regarding RNA splicing. Proteins involved in RNA splicing mechanisms were upregulated in those patients that received immunotherapy, with a lack of tumor response to treatment (i.e., started to progress after a few months). Among proteins with an important role in the alternative splicing processes, we can highlight the significantly upregulated SNRPB2, SNRNP70 and SNRPA1 proteins [90] (GO Biological Process, FDR < 0.05) (Figure S4) (Figure 6 2D). Studies have confirmed that the abnormal expression of the splicing proteins can lead to inappropriate splicing mechanisms, causing tumor progression [91]. Denga et al. also suggested that these disordered splicing mechanisms could promote the loss of cell surface antigens, which are crucial in melanoma and immune cell fusion that might contribute to immunotherapy resistance [91]. In the clinical field, the anti-spliceosome therapies are currently debated in their application of spliceosome machinery targeting [92]. There are several investigations on small molecules which inhibit the steps of the splicing mechanisms, but the usefulness and clinical safety of these approaches are still under investigation [92,93]. Our results can support the importance of studies that identify anti-spliceosome drugs and can provide insight into the protein mechanisms related to immunotherapy resistance. 
1,

A)

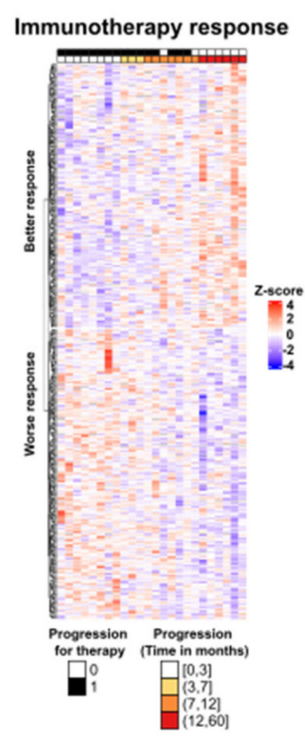

Protein and pathway expression pattern in the therapy response subgroups

Pathways of the subgroups
B)

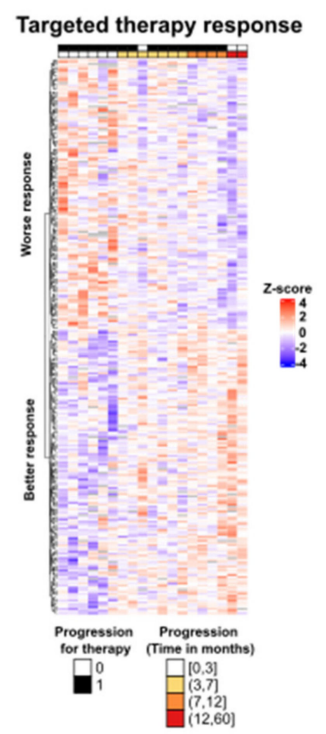

2,

C)

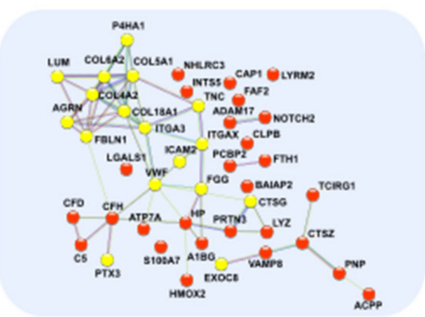

E)

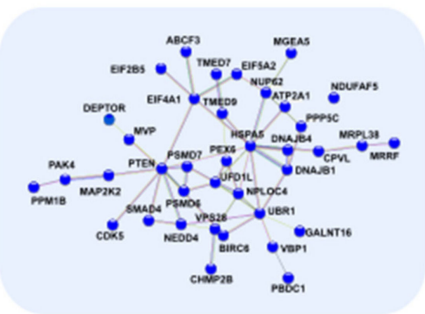

D)

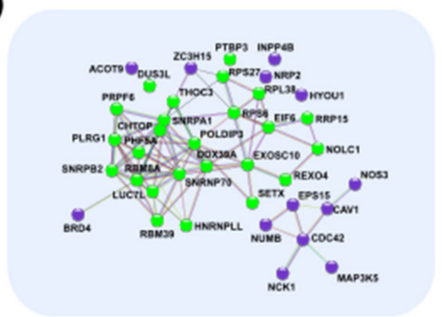

F)

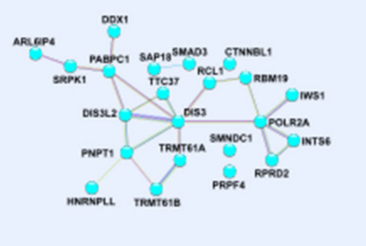

Figure 6. The heatmap of the treatment subgroups and the highlighted pathways. (1) Protein and pathway expression pattern in the therapy response subgroups. (A) Heatmap of the up- and downregulated proteins in patients with better and worse response in the immunotherapy subgroup. (B) Heatmap of the up- and downregulated proteins in patients with better and worse response in the targeted therapy subgroup. (2) Protein-protein interaction network of the up- and downregulated proteins in each treatment subgroup. (C) Proteins upregulated in patients with better outcome in the immunotherapy subgroup. The yellow color shows the extracellular matrix components, and red indicates the components from the immune system. (D) Proteins upregulated in patients with worse response in the immunotherapy subgroup. Proteins from the RNA processing are highlighted in green, whereas the purple color shows the members of the VEGFA-VEGFR2 pathway. (E) Proteins upregulated in patients with worse outcomes in the targeted therapy subgroup. The pathway analysis highlighted the key role of proteins involved in metabolic pathways. (F) Proteins upregulated in patients with better outcome in the targeted therapy subgroup. Multiple elements of RNA processing can be detected. The list of proteins involved in the aforementioned pathways is given in the Supplementary Document S1. 
3.8.2. Proteins and Pathways Indicating Better Outcome in the Immunotherapy-Treated Subgroup

We also identified proteins that are potential predictors of improved response to immunotherapy (Figure $6(2 \mathrm{C})$ ). In this subgroup of proteins, the stroma-induced signatures and the components of the immune system were significantly overrepresented. In the subgroup with better response to immunotherapy, the significantly upregulated proteins and pathways were enriched in functions such as neutrophil degranulation, complement cascade, B-cell differentiation, neutrophil-mediated immunity, extracellular (EC) organization, ECM-receptor interaction, (for example, ARGN protein), cell adhesion (for instance, ICAM2 protein), integrin cell surface interactions and the PI3K-Akt pathway, the components of which are related to cell adhesion (represented by proteins such as COL4A2 and COL6A2) (KEGG pathways, GO biological processes, FDR < 0.05) (Figure 6 (2C)). In our study, those proteins that were incorporated in the neutrophil degranulation, such as proteinase 3 (PRTN3) [94,95], showed an upregulated pattern aligned with the slow and late development of the progressive behavior of the tumor with a better outcome. In agreement with our results, Babačić et al. revealed that during anti-PD-1-R therapy, increased neutrophil degranulation was detected in the plasma and consequently the neutrophil to lymphocyte ratio was correlated to the longer overall survival in this in-depth plasma proteomics study [96]. Besides the immune response, the function of the EC organization in the tumor microenvironment is not negligible. There are several studies that highlight the coupled functions of ECM and the immune system against melanoma. For instance, in a recent study, Fejza et al. presented that the ECM proteins are found to be associated with the efficacy of the PD-1 treatments [97]. Interestingly, in our study, the EC proteins were upregulated, mostly in cell adhesion components, in the late progressive phase of the tumor development and linked to the longer survival rate as well as the response to immunotherapy. Furthermore, the ECM serves as a basis of the pre-metastatic niche, which is a new and unidentified target regarding the anti-cancer therapies. A recent study summarized those mechanisms that can be crucial in preventing the development of "the metastatic soils" leading to arrest of the tumor invasion [98]. For instance, the prevention of EC vesicles such CXCR2 and CXCR4 inhibitors, vascular stabilization and the immunomodulation can lead to the demolition of the complicated mechanisms in the stroma signature [98]. Further investigation is needed to strengthen the role of both the ECM's and the microenvironment's role in tumor management.

\subsubsection{Proteins and Pathways Altering the Patient Outcome in the Targeted Therapy Subgroup}

The responsiveness to the targeted therapy can arise from abundant mechanisms, such as the upregulation of survival pathways, reactivation of MAPK or the presence of a dedifferentiated/MITFlow cell state [99]. In our functional analysis of proteins that indicate worse prognosis by their overexpression, the metabolic pathways were significantly enriched (KEGG pathways, GO biological processes: FDR < 0.05) (Figure $6(2 \mathrm{E})$ ). Specifically, the MAPK signaling pathway elements (such as PAK4 and MAP2K2 proteins), mTOR pathway members (such as PTEN and DEPTOR proteins), heat shock response proteins and cyclin-dependent kinase 5 were enriched to the same extent in the metabolic pathways and implicated in the resistance mechanisms in the targeted therapy subgroup in our pilot study. The reactivation of the MAPK pathway is reported in up to $60 \%$ of melanoma [99-102], intrinsically linked to our results, in which the proteins concerning the MAPK pathway showed upregulation in the early progressors in the targeted therapy subgroup. Proteins pertaining to the PTEN and mTOR signaling pathways, also implicated worse outcomes during the administration of targeted therapy. These findings correspond to several studies, demonstrating that the activation of mTOR accelerates the proliferation and survival mechanisms of the melanoma cell, thus leading to resistance to BRAF inhibitors $[99,103,104]$. Genetic alterations of PTEN can be found in $30 \%$ of melanomas and also are associated with the BRAF V600E mutations [99,105-107]. As a consequence of these genetic changes, the upregulation of the PI3K-PTEN-Akt might promote the resistance 
mechanisms to targeted therapies in some cases [108]. Intriguingly, in our patient cohort, the PTEN expression pattern was also the strongest in three patients whose tumor progressed in five months alongside targeted therapy. Clinicopathologic similarities were also observed in these three cases. The Breslow level of these primary melanomas were more than $4 \mathrm{~mm}$ with histopathologic classification of pT4 (AJCC8). All of these were nodular melanomas with a short progression-free survival. The behavior of these three melanomas can be described as aggressive in parallel with the fast progression rate throughout the therapy. These mechanisms may contribute to the complicated resistance mechanisms in melanoma during treatments and to ascertaining the hidden effect of the metabolic pathways. To verify these results, further investigations including a larger patient cohort are needed.

In the functional analysis of predictive proteins providing better response to targeted therapy, we found significantly up- and downregulated pathways such as mRNA processing, RNA degradation, metabolism of RNA and proteins referring to spliceosomes (KEGG pathways, GO biological processes, FDR < 0.05) (Figure 62 F). Intriguingly, the RNA splicing mechanisms were also observed in the analysis of the immunotherapy subgroup, but there, activation of these processes was linked to worse prognosis. Our data thus suggests the ambivalent role the RNA mechanisms potentially might have: (i) acting as predictors of better response in patients receiving targeted therapy; (ii) predictors of worse response in patients receiving immunotherapy. However, it is noteworthy that the proteins involved in RNA mechanisms in the targeted therapy subgroup are not identical to the ones within the immunotherapy subgroup. Moreover, we have seen a downregulation of proteins in cell adhesion and ECM organization in early progressed tumors, an observation also made in the immunotherapy subgroups. Thus, our results indicate that the overexpression of the proteins in EC mechanisms can be associated with good prognosis and may predict a better therapy response. These data may raise questions about the real role of the stroma in the melanoma response during the application of certain treatments.

We acknowledge that our metastatic cohort study has some limitations, such as the heterogeneity of the samples due to the different histotypes with varying histopathologic characterization and clinical parameters. Furthermore, tumor content of the samples involved in the therapy subgroups are ranging from a few percent up to $90 \%$, which means that significant mechanisms incorporating therapy response can be related to pathways in stroma cells. The upregulation of EC components in some samples gives us hints about the effect of tumor content differences on the proteomic profiles. On the other hand, our findings can form the basis of further investigations highlighting the role of the tumor-stroma interactions on the progression of the melanoma.

\section{Conclusions}

As melanoma malignum constitutes a highly heterogeneous tumor morphology, diagnosing and high precision accuracy are major challenges.

Beyond the well-known predictive proteins such as mutant BRAF kinase and plasma lactate dehydrogenase (LDH), which are used on a daily basis worldwide, additional protein biomarkers are necessary to unravel resistance mechanisms and predicting the efficacy of the treatments.

The proteomic analysis of 90 FFPE melanoma samples revealed the discovery of predictive protein candidates related to the progression-free survival as well as the therapy responsiveness. The histopathologic analysis, clinical parameters of the patient cohort and the expression pattern of the proteins were outlined. We found that certain mechanisms and protein expressions in melanoma aligned with disease progression. For instance, in sample clusters with enrichment for shorter survival, TRAF6, ARMC10, CDK4, ITGA5, CAMK4 and WIPI proteins were highly expressed, and AIF1 and PPIF proteins were lowly expressed. The outlined proteins can be further investigated for their involvement in melanoma progression. The expression pattern of the keratinization pathways was different in the comparison of primary and metastasis samples. Furthermore, the upregulation of 
CD46, MCM9, and TRAF1 proteins and the downregulation of proteins such as PELO and PSMD3 was identified in connection with clinical stages (from AJCC8 stage II. to IV.).

On the basis of the therapy subgroups investigated, a new role of well recognized pathways and proteins in melanoma development was recognized in the therapy response. In our findings, the VEGFA-VEGFR2 pathway (such as NOS3 protein) and RNA splicing mechanism were related to a worse prognosis, and neutrophil degranulation mechanisms and extracellular matrix interactions were connected to better survival during immunotherapy treatments. Furthermore, the upregulation of the MAPK and mTOR signaling components (such as PTEN protein) was associated with short survival, and the significant activation of the RNA mechanisms and stroma signature were connected to better outcome under targeted therapy. These results elucidate new clinical avenues in the underlying molecular mechanisms during the evolution of therapy resistance. Our findings also manifested the essential role regarding the immune system, VEGF inhibitors, spliceosome inhibitors and stroma targets in melanoma treatment. In the coming years, the emphasis will be on discovering and developing predictive biomarkers with hidden and unpredictive melanoma mechanisms during ongoing patient treatments, because this is a strategy to strengthen the immunotherapy response, thereby lowering the impact of developing novel disease mechanisms leading to tumor progression.

Taken together, a broad spectrum of mechanisms involved in tumor progression and therapy response were demonstrated within our study, verifying the complexity of the potentially predictive protein biomarkers in melanoma.

Supplementary Materials: The following are available online at https:/ / www.mdpi.com/article/ 10.3390/cancers13236105/s1, Figure S1: Quality control plots for proteomic data normalization and batch effect correction, Figure S2: Addressing sample heterogeneity in our study, Figure S3: Kaplan-Meier Survival analysis of the treated patients based on their AJCC8 classification stage during disease-free survival, Figure S4: Functional annotation (Gene Ontology Biological Processes) of proteins significantly (FDR $<0.05$ ) associated with therapy response, Table S1: Fisher's exact test results for clinical and histopathological categories (borderline) significantly enriched in the detected sample clusters, Table S2: The stages of patients at diagnosis and after follow-up in Cluster 1, 2 and 4. Clinical stage based on AJCC8 classification. Supplementary Document S1: All statistics of the proteomic data, the summarized proteomics data, GSEA results for prim vs. met, GSEA results for clinical stages and the proteins and pathways of the therapy subgroups labeled on Figure 6(2).

Author Contributions: Conceptualization, L.S., E.V., I.B.N.; Methodology, L.S., E.V., I.B.N., B.S., N.P.d.A., H.O., M.M.-V., P.H.; Validation, L.S., E.V., I.B.N.; Formal Analysis, B.S., P.H.; Investigation, L.S., E.V., M.d.C.B.-A.; Funding acquisition, I.B.N., G.M.-V.; Project administration, L.S., I.B.N., G.M.-V.; Supervision, I.B.N., G.M.-V., L.K., G.D., A.M.S.; Writing—original draft, L.S., E.V., B.S., J.G., L.H.B., I.B.N., G.M.-V.; Writing-review and editing, L.H.B., B.S., Á.J.J., I.B.N., G.M.-V., B.B., J.M., P.H., L.K., G.D. All authors have read and agreed to the published version of the manuscript.

Funding: This study was supported by grants from Berta Kamprad Foundation (The Impact of Melanoma Tumor Heterogeneity on Drug Treatment Effects, 2021-004), Lund, Sweden, as well as from GINOP-2.3.2-15-2016-00020 TUMORDNS (N.I.B.) (L.S.) and K125509 grant from the National Academy of Sciences (N.I.B.), and supported by the ÚNKP-21-3-SZTE-102 New National Excellence Program of the Ministry for Innovation and Technology from the source of the National Research, Development and Innovation Fund (University of Szeged, Szeged, Hungary) (L.S.).

Institutional Review Board Statement: This study was conducted according to the guidelines of the Declarations of Helsinki and approved by the Hungarian Ministry of Human Resources, Deputy State Secretary for National Chief Medical Officer, Department of Health Administration. The protocol code is MEL-PROTEO-001, the approval number is 4463-6/2018/EÜIG and the date of approval is 12 March 2018.

Informed Consent Statement: Due to the retrospective anonymized FFPE samples, informed consent was not applicable, referring to the MEL-PROTEO-001, 4463-6/2018/EÜIG ethical approval. 
Data Availability Statement: ANOVA and Tukey's Honest Significant Difference test results of sample cluster comparisons, as well as Cox regression analysis results for immunotherapy and targeted therapy subgroups, proteomic data summary, $t$-test results (paired/independent) for primary vs. metastasis comparisons as well as ANCOVA results for the comparison of disease stages are provided in Supplementary Document S1. The scripts used for the proteomic data normalization, batch effect correction and statistics are available at https://github.com/bszeitz/MM_pilot (accessed on 21 November 2021). The mass spectrometry proteomics data have been deposited to the ProteomeXchange Consortium via the PRIDE [1] partner repository with the dataset identifier PXD028930. Project name is Proteomic Analysis on Paraffine Archived Melanoma. Project accession is PXD028930, project DOI is not applicable, username is reviewer_pxd028930@ebi.ac.uk, and password is TaHGkBGm. Clinical information of individual patients cannot be provided due to the ethics restrictions.

Acknowledgments: This study was generously sponsored by grants from the Berta Kamprad Foundation, Lund, Sweden. In addition, we would like to thank Thermo Fisher Scientific for their generous support and Liconic UK, for Biobanking support. This work was also made under the auspices of a Memorandum of Understanding between the European Cancer Moonshot Center in Lund and the U.S. National Cancer Institute's International Cancer Proteogenome Consortium (ICPC). ICPC encourages international cooperation among institutions and nations in proteogenomic cancer research in which proteogenomic datasets are made available to the public. In addition, the study was conducted in collaboration with the U.S. National Cancer Institute's Clinical Proteomic Tumor Analysis Consortium (CPTAC). The study was also conducted under the Memorandum of Understanding between the Federal University of Rio de Janeiro, Brazil (grants CAPES 88887.130697, CNPq 440613/2016-7 and 308341-2019-8, and FAPERJ E26/210.173/2018 to G.B.), and Lund University, Sweden. We thank the Brazilian foundation CNPq for the research scholarship.

Conflicts of Interest: The authors declare no conflict of interest.

\section{Abbreviations}

AIF1

apoptosis-inducing factor 1

Akt protein kinase $B$ (collective name of the serine/threonine-specific protein

ARGI1

ARGN

ARMC10

BRAF

CAMK4

CD33

CD46

CDK2

CHD8

CKIT

c-Myc

COL4A2

COL6A2

CR2

CSF1R

CXCR2

CXCR4

DEPTOR

ERK

FCERG

FDR

GIMA5

HCT-116

kinases (Akt1,2,3)

arginase- 1

isoform 6 of Agrin

armadillo repeat-containing protein 10

B-raf proto-oncogene, serine/threonine kinase

calcium/calmodulin dependent protein kinase IV

myeloid cell surface antigen CD33 (cluster of differentiation 33)

cluster of differentiation 46

cyclin-dependent kinase 2

Chromodomain-helicase-DNA-binding protein 8

KIT proto-oncogene, receptor tyrosine kinase

MYC proto-oncogene, bHLH transcription factor

collagen type IV alpha 2 chain

collagen type VI alpha 2 chain

complement C3d receptor 2

Colony stimulating factor 1 receptor

C-X-C motif chemokine receptor 2

C-X-C motif chemokine receptor 4

DEP domain containing mTOR interacting protein

extracellular signal regulated kinases, classical MAP kinases

fusion protein erythroblast transformation specific related gene

False Discovery Rate

GTPase IMAP family member 5

human colorectal carcinoma cell line

HT-29

human colon cancer cell line 


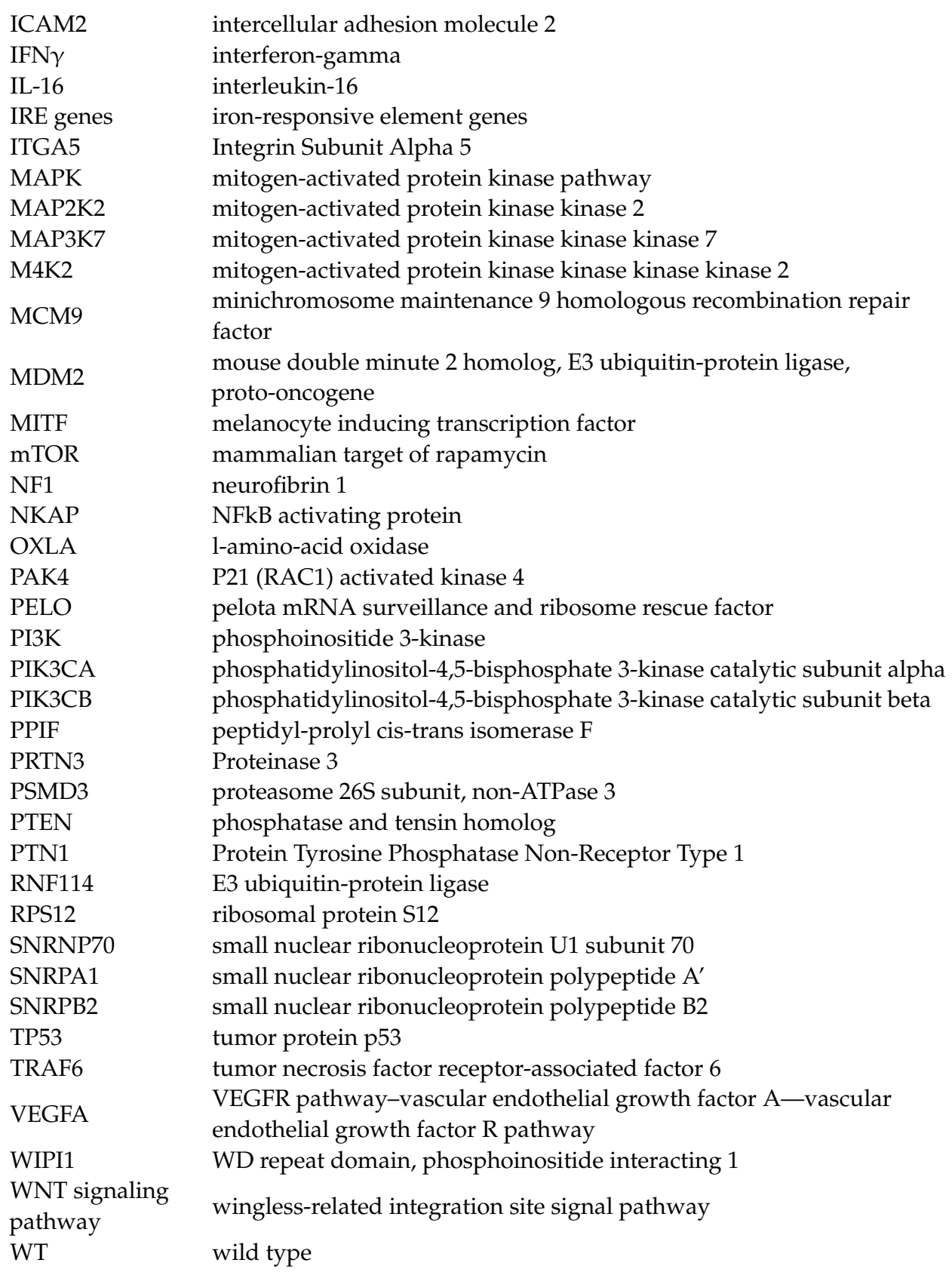

\section{References}

1. Tímár, J.; Vizkeleti, L.; Doma, V.; Barbai, T.; Rásó, E. Genetic Progression of Malignant Melanoma. Cancer Metastasis Rev. 2016, 35, 93-107. [CrossRef] [PubMed]

2. Boussios, S.; Rassy, E.; Samartzis, E.; Moschetta, M.; Sheriff, M.; Pérez-Fidalgo, J.A.; Pavlidis, N. Melanoma of Unknown Primary: New Perspectives for an Old Story. Crit. Rev. Oncol. Hematol. 2021, 158, 103208. [CrossRef] [PubMed]

3. Cancer Today. Available online: http://gco.iarc.fr/today/home (accessed on 24 November 2021).

4. Schadendorf, D.; van Akkooi, A.C.J.; Berking, C.; Griewank, K.G.; Gutzmer, R.; Hauschild, A.; Stang, A.; Roesch, A.; Ugurel, S. Melanoma. Lancet 2018, 392, 971-984. [CrossRef]

5. Thomas, D.; Bello, D.M. Adjuvant Immunotherapy for Melanoma. J. Surg. Oncol. 2021, 123, 789-797. [CrossRef] [PubMed]

6. Garbe, C.; Amaral, T.; Peris, K.; Hauschild, A.; Arenberger, P.; Bastholt, L.; Bataille, V.; del Marmol, V.; Dréno, B.; Fargnoli, M.C.; et al. European Consensus-Based Interdisciplinary Guideline for Melanoma. Part 2: Treatment-Update 2019. Eur. J. Cancer 2020, 126, 159-177. [CrossRef] [PubMed]

7. Betancourt, L.H.; Szasz, A.M.; Kuras, M.; Rodriguez Murillo, J.; Sugihara, Y.; Pla, I.; Horvath, Z.; Pawłowski, K.; Rezeli, M.; Miharada, K.; et al. The Hidden Story of Heterogeneous B-Raf V600E Mutation Quantitative Protein Expression in Metastatic Melanoma-Association with Clinical Outcome and Tumor Phenotypes. Cancers 2019, 11, 1981. [CrossRef] [PubMed] 
8. Boni, A.; Cogdill, A.P.; Dang, P.; Udayakumar, D.; Njauw, C.-N.J.; Sloss, C.M.; Ferrone, C.R.; Flaherty, K.T.; Lawrence, D.P.; Fisher, D.E.; et al. Selective BRAFV600E Inhibition Enhances T-Cell Recognition of Melanoma without Affecting Lymphocyte Function. Cancer Res. 2010, 70, 5213-5219. [CrossRef]

9. Liu, C.; Peng, W.; Xu, C.; Lou, Y.; Zhang, M.; Wargo, J.A.; Chen, J.Q.; Li, H.S.; Watowich, S.S.; Yang, Y.; et al. BRAF Inhibition Increases Tumor Infiltration by $\mathrm{T}$ Cells and Enhances the Antitumor Activity of Adoptive Immunotherapy in Mice. Clin. Cancer Res. 2013, 19, 393-403. [CrossRef]

10. Ebert, P.J.R.; Cheung, J.; Yang, Y.; McNamara, E.; Hong, R.; Moskalenko, M.; Gould, S.E.; Maecker, H.; Irving, B.A.; Kim, J.M.; et al. MAP Kinase Inhibition Promotes T Cell and Anti-Tumor Activity in Combination with PD-L1 Checkpoint Blockade. Immunity 2016, 44, 609-621. [CrossRef]

11. Herbst, R.S.; Soria, J.-C.; Kowanetz, M.; Fine, G.D.; Hamid, O.; Gordon, M.S.; Sosman, J.A.; McDermott, D.F.; Powderly, J.D.; Gettinger, S.N.; et al. Predictive Correlates of Response to the Anti-PD-L1 Antibody MPDL3280A in Cancer Patients. Nature 2014, 515, 563-567. [CrossRef]

12. Cancer Genome Atlas Network Genomic Classification of Cutaneous Melanoma. Cell 2015, 161, 1681-1696. [CrossRef]

13. Velasquez, E.; Szadai, L.; Zhou, Q.; Kim, Y.; Pla, I.; Sanchez, A.; Appelqvist, R.; Oskolas, H.; Marko-Varga, M.; Lee, B.; et al. A Biobanking Turning-Point in the Use of Formalin-Fixed, Paraffin Tumor Blocks to Unveil Kinase Signaling in Melanoma. Clin. Transl. Med. 2021, 11, e466. [CrossRef]

14. Čuklina, J. Computational Challenges in Biomarker Discovery from High-Throughput Proteomic Data. Ph.D. Thesis, ETH Zurich, Zurich, Switzerland, 2018.

15. Wickham, H. Ggplot2: Elegant Graphics for Data Analysis; Springer: New York, NY, USA, 2016; ISBN 978-3-319-24275-0.

16. Vu, V. Ggbiplot. Available online: https:/ / github.com/vqv/ggbiplot (accessed on 24 November 2021).

17. Wilke, C.O. Cowplot: Streamlined Plot Theme and Plot Annotations for "Ggplot2". Available online: https://CRAN.R-project. org / package $=$ cowplot (accessed on 24 November 2021).

18. Auguie, B.; Antonov, A. GridExtra: Miscellaneous Functions for “Grid” Graphics. Available online: https://CRAN.R-project. org / package=gridExtra (accessed on 24 November 2021).

19. Gu, Z.; Eils, R.; Schlesner, M. Complex Heatmaps Reveal Patterns and Correlations in Multidimensional Genomic Data. Bioinformatics 2016, 32, 2847-2849. [CrossRef]

20. Langfelder, P.; Zhang, B.; Horvath, S. Defining Clusters from a Hierarchical Cluster Tree: The Dynamic Tree Cut Package for R. Bioinformatics 2008, 24, 719-720. [CrossRef]

21. Kanehisa, M.; Goto, S. KEGG: Kyoto Encyclopedia of Genes and Genomes. Nucleic Acids Res. 2000, 28, 27-30. [CrossRef]

22. Jassal, B.; Matthews, L.; Viteri, G.; Gong, C.; Lorente, P.; Fabregat, A.; Sidiropoulos, K.; Cook, J.; Gillespie, M.; Haw, R.; et al. The Reactome Pathway Knowledgebase. Nucleic Acids Res. 2020, 48, D498-D503. [CrossRef]

23. ClusterProfiler 4.0: A Universal Enrichment Tool for Interpreting Omics Data-ScienceDirect. Available online: https://www. sciencedirect.com/science/article/pii/S2666675821000667 (accessed on 24 November 2021).

24. Gene Set Enrichment Analysis: A Knowledge-Based Approach for Interpreting Genome-Wide Expression Profiles I PNAS. Available online: https:/ / www.pnas.org/content/102/43/15545 (accessed on 24 November 2021).

25. Liberzon, A.; Subramanian, A.; Pinchback, R.; Thorvaldsdóttir, H.; Tamayo, P.; Mesirov, J.P. Molecular Signatures Database (MSigDB) 3.0. Bioinformatics 2011, 27, 1739-1740. [CrossRef]

26. Martens, M.; Ammar, A.; Riutta, A.; Waagmeester, A.; Slenter, D.N.; Hanspers, K.; Miller, R.; Digles, D.; Lopes, E.N.; Ehrhart, F.; et al. WikiPathways: Connecting Communities. Nucleic Acids Res. 2021, 49, D613-D621. [CrossRef]

27. Szklarczyk, D.; Gable, A.L.; Nastou, K.C.; Lyon, D.; Kirsch, R.; Pyysalo, S.; Doncheva, N.T.; Legeay, M.; Fang, T.; Bork, P.; et al. The STRING Database in 2021: Customizable Protein-Protein Networks, and Functional Characterization of User-Uploaded Gene/Measurement Sets. Nucleic Acids Res. 2021, 49, D605-D612. [CrossRef]

28. Gene Ontology Consortium the Gene Ontology Resource: Enriching a GOld Mine. Nucleic Acids Res. 2021, 49, D325-D334. [CrossRef]

29. Shannon, P.; Markiel, A.; Ozier, O.; Baliga, N.S.; Wang, J.T.; Ramage, D.; Amin, N.; Schwikowski, B.; Ideker, T. Cytoscape: A Software Environment for Integrated Models of Biomolecular Interaction Networks. Genome Res. 2003, 13, 2498-2504. [CrossRef] [PubMed]

30. Keung, E.Z.; Gershenwald, J.E. The Eighth Edition American Joint Committee on Cancer (AJCC) Melanoma Staging System: Implications for Melanoma Treatment and Care. Expert Rev. Anticancer Ther. 2018, 18, 775-784. [CrossRef] [PubMed]

31. Elder, D.E.; Massi, D.; Scolyer, R.A.; Willemze, R. WHO Classification of Skin Tumours; IARC: Lyon, France, 2018; ISBN 92-832-2440-X.

32. Dusek, R.L.; Attardi, L.D. Desmosomes: New Perpetrators in Tumour Suppression. Nat. Rev. Cancer 2011, 11, 317-323. [CrossRef] [PubMed]

33. Zhou, G.; Yang, L.; Gray, A.; Srivastava, A.K.; Li, C.; Zhang, G.; Cui, T. The Role of Desmosomes in Carcinogenesis. OncoTargets Ther. 2017, 10, 4059-4063. [CrossRef]

34. Valenzuela-Iglesias, A.; Burks, H.E.; Arnette, C.R.; Yalamanchili, A.; Nekrasova, O.; Godsel, L.M.; Green, K.J. Desmoglein 1 Regulates Invadopodia by Suppressing EGFR/Erk Signaling in an Erbin-Dependent Manner. Mol. Cancer Res. 2019, 17, 1195-1206. [CrossRef] 
35. Peitsch, W.K.; Doerflinger, Y.; Fischer-Colbrie, R.; Huck, V.; Bauer, A.T.; Utikal, J.; Goerdt, S.; Schneider, S.W. Desmoglein 2 Depletion Leads to Increased Migration and Upregulation of the Chemoattractant Secretoneurin in Melanoma Cells. PLoS ONE 2014, 9, e89491. [CrossRef]

36. Hütz, K.; Zeiler, J.; Sachs, L.; Ormanns, S.; Spindler, V. Loss of Desmoglein 2 Promotes Tumorigenic Behavior in Pancreatic Cancer Cells. Mol. Carcinog. 2017, 56, 1884-1895. [CrossRef]

37. Lee, S.-H.; Kim, J.-M.; Lee, D.G.; Lee, J.; Park, J.-G.; Han, T.-S.; Cho, H.-S.; Cho, Y.-L.; Bae, K.-H.; Park, Y.-J.; et al. Loss of Desmoglein-2 Promotes Gallbladder Carcinoma Progression and Resistance to EGFR-Targeted Therapy through Src Kinase Activation. Cell Death Differ. 2021, 28, 968-984. [CrossRef]

38. Tan, L.Y.; Mintoff, C.; Johan, M.Z.; Ebert, B.W.; Fedele, C.; Zhang, Y.F.; Szeto, P.; Sheppard, K.E.; McArthur, G.A.; Foster-Smith, E.; et al. Desmoglein 2 Promotes Vasculogenic Mimicry in Melanoma and Is Associated with Poor Clinical Outcome. Oncotarget 2016, 7, 46492-46508. [CrossRef]

39. Lo, A.K.; Yuen, P.W.; Liu, Y.; Wang, X.H.; Cheung, A.L.; Wong, Y.C.; Tsao, S.W. Downregulation of Hemidesmosomal Proteins in Nasopharyngeal Carcinoma Cells. Cancer Lett. 2001, 163, 117-123. [CrossRef]

40. Kim, H.J.; Maiti, P.; Barrientos, A. Mitochondrial Ribosomes in Cancer. Semin. Cancer Biol. 2017, 47, 67-81. [CrossRef]

41. Huang, G.; Li, H.; Zhang, H. Abnormal Expression of Mitochondrial Ribosomal Proteins and Their Encoding Genes with Cell Apoptosis and Diseases. Int. J. Mol. Sci. 2020, 21, 8879. [CrossRef]

42. Liu, J.; Wu, S.; Xie, X.; Wang, Z.; Lei, Q. Identification of Potential Crucial Genes and Key Pathways in Osteosarcoma. Hereditas 2020, 157, 29. [CrossRef]

43. Tang, N.-Y.; Chueh, F.-S.; Yu, C.-C.; Liao, C.-L.; Lin, J.-J.; Hsia, T.-C.; Wu, K.-C.; Liu, H.-C.; Lu, K.-W.; Chung, J.-G. Benzyl Isothiocyanate Alters the Gene Expression with Cell Cycle Regulation and Cell Death in Human Brain Glioblastoma GBM 8401 Cells. Oncol. Rep. 2016, 35, 2089-2096. [CrossRef]

44. Sotgia, F.; Fiorillo, M.; Lisanti, M.P. Mitochondrial Markers Predict Recurrence, Metastasis and Tamoxifen-Resistance in Breast Cancer Patients: Early Detection of Treatment Failure with Companion Diagnostics. Oncotarget 2017, 8, 68730-68745. [CrossRef]

45. Federico, A.; Rienzo, M.; Abbondanza, C.; Costa, V.; Ciccodicola, A.; Casamassimi, A. Pan-Cancer Mutational and Transcriptional Analysis of the Integrator Complex. Int. J. Mol. Sci. 2017, 18, 936. [CrossRef]

46. Yadavilli, S.; Mayo, L.D.; Higgins, M.; Lain, S.; Hegde, V.; Deutsch, W.A. Ribosomal Protein S3: A Multi-Functional Protein That Interacts with Both P53 and MDM2 through Its KH Domain. DNA Repair 2009, 8, 1215-1224. [CrossRef]

47. Murray, L.B.; Lau, Y.-K.I.; Yu, Q. Merlin Is a Negative Regulator of Human Melanoma Growth. PLoS ONE 2012 , 7, e43295. [CrossRef]

48. Pio, R.; Corrales, L.; Lambris, J.D. The Role of Complement in Tumor Growth. Adv. Exp. Med. Biol. 2014, 772, 229-262. [CrossRef]

49. Zhang, R.; Liu, Q.; Li, T.; Liao, Q.; Zhao, Y. Role of the Complement System in the Tumor Microenvironment. Cancer Cell Int. 2019, 19, 300. [CrossRef]

50. Simpson-Haidaris, P.J.; Rybarczyk, B. Tumors and Fibrinogen. The Role of Fibrinogen as an Extracellular Matrix Protein. Ann. N. Y. Acad. Sci. 2001, 936, 406-425. [CrossRef] [PubMed]

51. Sade-Feldman, M.; Kanterman, J.; Klieger, Y.; Ish-Shalom, E.; Olga, M.; Saragovi, A.; Shtainberg, H.; Lotem, M.; Baniyash, M. Clinical Significance of Circulating CD33+CD11b+HLA-DR- Myeloid Cells in Patients with Stage IV Melanoma Treated with Ipilimumab. Clin. Cancer Res. 2016, 22, 5661-5672. [CrossRef] [PubMed]

52. Choi, J.W.; Kim, Y.J.; Yun, K.A.; Won, C.H.; Lee, M.W.; Choi, J.H.; Chang, S.E.; Lee, W.J. The Prognostic Significance of VISTA and CD33-Positive Myeloid Cells in Cutaneous Melanoma and Their Relationship with PD-1 Expression. Sci. Rep. 2020, 10, 14372. [CrossRef] [PubMed]

53. Grzywa, T.M.; Sosnowska, A.; Matryba, P.; Rydzynska, Z.; Jasinski, M.; Nowis, D.; Golab, J. Myeloid Cell-Derived Arginase in Cancer Immune Response. Front. Immunol. 2020, 11, 938. [CrossRef]

54. Pajerowski, A.G.; Nguyen, C.; Aghajanian, H.; Shapiro, M.J.; Shapiro, V.S. NKAP Is a Transcriptional Repressor of Notch Signaling and Is Required for T Cell Development. Immunity 2009, 30, 696-707. [CrossRef]

55. Shu, W.; Liu, G.; Dai, Y.; Feng, A.; Chen, Z.; Han, J.; Li, X. The Oncogenic Role of NKAP in the Growth and Invasion of Colon Cancer Cells. Oncol. Rep. 2019, 42, 2130-2138. [CrossRef]

56. Liu, J.; Zhang, M.; Kan, Y.; Wang, W.; Liu, J.; Gong, J.; Yang, J. Nuclear Factor-KB Activating Protein Plays an Oncogenic Role in Neuroblastoma Tumorigenesis and Recurrence Through the Phosphatidylinositol 3-Kinase/Protein Kinase B Signaling Pathway. Front. Cell Dev. Biol. 2020, 8, 622793. [CrossRef]

57. Patterson, A.R.; Endale, M.; Lampe, K.; Aksoylar, H.I.; Flagg, A.; Woodgett, J.R.; Hildeman, D.; Jordan, M.B.; Singh, H.; Kucuk, Z.; et al. Gimap5-Dependent Inactivation of GSK3 $\beta$ Is Required for CD4 + T Cell Homeostasis and Prevention of Immune Pathology. Nat. Commun. 2018, 9, 430. [CrossRef]

58. Patterson, A.R.; Bolcas, P.; Lampe, K.; Cantrell, R.; Ruff, B.; Lewkowich, I.; Hogan, S.P.; Janssen, E.M.; Bleesing, J.; Khurana Hershey, G.K.; et al. Loss of GTPase of Immunity-Associated Protein 5 (Gimap5) Promotes Pathogenic CD4+ T-Cell Development and Allergic Airway Disease. J. Allergy Clin. Immunol. 2019, 143, 245-257.e6. [CrossRef]

59. Dai, P.; Tang, Z.; Ruan, P.; Bajinka, O.; Liu, D.; Tan, Y. Gimap5 Inhibits Lung Cancer Growth by Interacting With M6PR. Front. Oncol. 2021, 11, 699847. [CrossRef]

60. Chuang, H.-C.; Wang, X.; Tan, T.-H. MAP4K Family Kinases in Immunity and Inflammation. Adv. Immunol. 2016, 129, 277-314. [CrossRef] 
61. Jauliac, S.; Mazerolles, F.; Jabado, N.; Pallier, A.; Bernard, F.; Peake, J.; Fischer, A.; Hivroz, C. Ligands of CD4 Inhibit the Association of Phospholipase Cgamma1 with Phosphoinositide 3 Kinase in T Cells: Regulation of This Association by the Phosphoinositide 3 Kinase Activity. Eur. J. Immunol. 1998, 28, 3183-3191. [CrossRef]

62. Whale, A.D.; Colman, L.; Lensun, L.; Rogers, H.L.; Shuttleworth, S.J. Functional Characterization of a Novel Somatic Oncogenic Mutation of PIK3CB. Signal Transduct. Target. Ther. 2017, 2, 17063. [CrossRef]

63. Lin, C.-W.; Lai, G.-M.; Chen, K.-C.; Lin, T.-H.; Fan, J.-J.; Hsu, R.-L.; Chou, C.-M.; Lin, C.-M.; Kandaswami, C.C.; Lee, M.-T.; et al. RPS12 Increases the Invasiveness in Cervical Cancer Activated by C-Myc and Inhibited by the Dietary Flavonoids Luteolin and Quercetin. J. Funct. Foods 2015, 19, 236-247. [CrossRef]

64. Sadik, A.; Somarribas Patterson, L.F.; Öztürk, S.; Mohapatra, S.R.; Panitz, V.; Secker, P.F.; Pfänder, P.; Loth, S.; Salem, H.; Prentzell, M.T.; et al. IL4I1 Is a Metabolic Immune Checkpoint That Activates the AHR and Promotes Tumor Progression. Cell 2020, 182, 1252-1270.e34. [CrossRef]

65. Wright, K.D.; Miller, B.S.; El-Meanawy, S.; Tsaih, S.-W.; Banerjee, A.; Geurts, A.M.; Sheinin, Y.; Sun, Y.; Kalyanaraman, B.; Rui, H.; et al. The P52 Isoform of SHC1 Is a Key Driver of Breast Cancer Initiation. Breast Cancer Res. 2019, 21, 74. [CrossRef]

66. Luo, Z.; Zhang, X.; Zeng, W.; Su, J.; Yang, K.; Lu, L.; Lim, C.B.; Tang, W.; Wu, L.; Zhao, S.; et al. TRAF6 Regulates Melanoma Invasion and Metastasis through Ubiquitination of Basigin. Oncotarget 2016, 7, 7179-7192. [CrossRef]

67. Lin, B.; Ke, Q.; Leaman, D.W.; Goel, V.; Agarwal, A. Regulation of RANKL-Induced Osteoclastogenesis by RING Finger Protein RNF114. J. Orthop. Res. 2018, 36, 159-166. [CrossRef]

68. Landström, M. The TAK1-TRAF6 Signalling Pathway. Int. J. Biochem. Cell Biol. 2010, 42, 585-589. [CrossRef]

69. Du, J.; Widlund, H.R.; Horstmann, M.A.; Ramaswamy, S.; Ross, K.; Huber, W.E.; Nishimura, E.K.; Golub, T.R.; Fisher, D.E. Critical Role of CDK2 for Melanoma Growth Linked to Its Melanocyte-Specific Transcriptional Regulation by MITF. Cancer Cell 2004, 6, 565-576. [CrossRef]

70. Desai, B.M.; Villanueva, J.; Nguyen, T.-T.K.; Lioni, M.; Xiao, M.; Kong, J.; Krepler, C.; Vultur, A.; Flaherty, K.T.; Nathanson, K.L.; et al. The Anti-Melanoma Activity of Dinaciclib, a Cyclin-Dependent Kinase Inhibitor, Is Dependent on P53 Signaling. PLoS ONE 2013, 8, e59588. [CrossRef]

71. Huang, R.; Xing, Z.; Luan, Z.; Wu, T.; Wu, X.; Hu, G. A Specific Splicing Variant of SVH, a Novel Human Armadillo Repeat Protein, Is up-Regulated in Hepatocellular Carcinomas. Cancer Res. 2003, 63, 3775-3782. [PubMed]

72. Zhou, X.; Yang, G.; Huang, R.; Chen, X.; Hu, G. SVH-B Interacts Directly with P53 and Suppresses the Transcriptional Activity of P53. FEBS Lett. 2007, 581, 4943-4948. [CrossRef] [PubMed]

73. Selimovic, D.; Sprenger, A.; Hannig, M.; Haïkel, Y.; Hassan, M. Apoptosis Related Protein-1 Triggers Melanoma Cell Death via Interaction with the Juxtamembrane Region of P75 Neurotrophin Receptor. J. Cell. Mol. Med. 2012, 16, 349-361. [CrossRef]

74. Shanmughapriya, S.; Rajan, S.; Hoffman, N.E.; Higgins, A.M.; Tomar, D.; Nemani, N.; Hines, K.J.; Smith, D.J.; Eguchi, A.; Vallem, S.; et al. SPG7 Is an Essential and Conserved Component of the Mitochondrial Permeability Transition Pore. Mol. Cell 2015, 60, 47-62. [CrossRef]

75. CD46 Protein Expression Summary-The Human Protein Atlas. Available online: https://www.proteinatlas.org/ENSG0000011 7335-CD46 (accessed on 24 November 2021).

76. Geller, A.; Yan, J. The Role of Membrane Bound Complement Regulatory Proteins in Tumor Development and Cancer Immunotherapy. Front. Immunol. 2019, 10, 1074. [CrossRef]

77. Cardone, J.; Le Friec, G.; Vantourout, P.; Roberts, A.; Fuchs, A.; Jackson, I.; Suddason, T.; Lord, G.; Atkinson, J.P.; Cope, A.; et al. Complement Regulator CD46 Temporally Regulates Cytokine Production by Conventional and Unconventional T Cells. Nat. Immunol. 2010, 11, 862-871. [CrossRef]

78. C, K.; Ac, C.; Jm, G.; Ka, B.; Km, M.; Jp, A. Activation of Human CD4+ Cells with CD3 and CD46 Induces a T-Regulatory Cell 1 Phenotype. Nature 2003, 421. [CrossRef]

79. Truscott, S.M.; Abate, G.; Price, J.D.; Kemper, C.; Atkinson, J.P.; Hoft, D.F. CD46 Engagement on Human CD4+ T Cells Produces T Regulatory Type 1-like Regulation of Antimycobacterial T Cell Responses. Infect. Immun. 2010, 78, 5295-5306. [CrossRef]

80. The Cancer Genome Atlas Program-National Cancer Institute. Available online: https://www.cancer.gov/about-nci/ organization/ccg/research/structural-genomics/tcga (accessed on 24 November 2021).

81. Han, W.; Wu, Y.-Z.; Zhao, X.-Y.; Gong, Z.-H.; Shen, G.-L. Integrative Analysis of Minichromosome Maintenance Proteins and Their Prognostic Significance in Melanoma. Front. Oncol. 2021, 11, 3203. [CrossRef]

82. PELO Pelota MRNA Surveillance and Ribosome Rescue Factor [Homo Sapiens (Human)]—Gene-NCBI. Available online: https:/ / www.ncbi.nlm.nih.gov / gene/53918 (accessed on 24 November 2021).

83. Patel, V.N.; Gokulrangan, G.; Chowdhury, S.A.; Chen, Y.; Sloan, A.E.; Koyutürk, M.; Barnholtz-Sloan, J.; Chance, M.R. Network Signatures of Survival in Glioblastoma Multiforme. PLoS Comput. Biol. 2013, 9, e1003237. [CrossRef]

84. Chen, J.; Wu, F.; Shi, Y.; Yang, D.; Xu, M.; Lai, Y.; Liu, Y. Identification of Key Candidate Genes Involved in Melanoma Metastasis. Mol. Med. Rep. 2019, 20, 903-914. [CrossRef]

85. Alessi, C.; Scapulatempo Neto, C.; Viana, C.R.; Vazquez, V. de L. PD-1/PD-L1 and VEGF-A/VEGF-C Expression in Lymph Node Microenvironment and Association with Melanoma Metastasis and Survival. Melanoma Res. 2017, 27, 565-572. [CrossRef]

86. Frezzetti, D.; Gallo, M.; Maiello, M.R.; D’Alessio, A.; Esposito, C.; Chicchinelli, N.; Normanno, N.; De Luca, A. VEGF as a Potential Target in Lung Cancer. Expert Opin. Ther. Targets 2017, 21, 959-966. [CrossRef] 
87. Ferrara, N.; Hillan, K.J.; Novotny, W. Bevacizumab (Avastin), a Humanized Anti-VEGF Monoclonal Antibody for Cancer Therapy. Biochem. Biophys. Res. Commun. 2005, 333, 328-335. [CrossRef]

88. VEGFA Protein Expression Summary-The Human Protein Atlas. Available online: https://www.proteinatlas.org/ENSG00000 112715-VEGFA (accessed on 24 November 2021).

89. De Melo, F.H.M.; Gonçalves, D.A.; de Sousa, R.X.; Icimoto, M.Y.; de Fernandes, D.C.; Laurindo, F.R.M.; Jasiulionis, M.G. Metastatic Melanoma Progression Is Associated with Endothelial Nitric Oxide Synthase Uncoupling Induced by Loss of ENOS:BH4 Stoichiometry. Int. J. Mol. Sci. 2021, 22, 9556. [CrossRef]

90. Wang, Y.; Liu, J.; Huang, B.; Xu, Y.-M.; Li, J.; Huang, L.-F.; Lin, J.; Zhang, J.; Min, Q.-H.; Yang, W.-M.; et al. Mechanism of Alternative Splicing and Its Regulation (Review). Biomed. Rep. 2015, 3, 152-158. [CrossRef]

91. Deng, K.; Yao, J.; Huang, J.; Ding, Y.; Zuo, J. Abnormal Alternative Splicing Promotes Tumor Resistance in Targeted Therapy and Immunotherapy. Transl. Oncol. 2021, 14, 101077. [CrossRef]

92. Eymin, B. Targeting the Spliceosome Machinery: A New Therapeutic Axis in Cancer? Biochem. Pharmacol. 2021, 189, 114039. [CrossRef]

93. Effenberger, K.A.; Urabe, V.K.; Jurica, M.S. Modulating Splicing with Small Molecular Inhibitors of the Spliceosome. Wiley Interdiscip. Rev. 2017, 8. [CrossRef]

94. Kuckleburg, C.J.; Newman, P.J. Neutrophil Proteinase 3 Acts on Protease-Activated Receptor-2 to Enhance Vascular Endothelial Cell Barrier Function. Arterioscler. Thromb. Vasc. Biol. 2013, 33, 275-284. [CrossRef] [PubMed]

95. Alatrash, G.; Vence, L.M.; Woodward, W.; Ueno, N.T.; Molldrem, J.J. Leukemia-Associated Primary Granule Proteins (PGPs) Elastase-2 and Proteinase-3 Are Aberrantly Expressed in Solid Tumors: A Potential Therapeutic Target for PR1-Directed Immunotherapy. Blood 2008, 112, 5440. [CrossRef]

96. Babačić, H.; Lehtiö, J.; Pico de Coaña, Y.; Pernemalm, M.; Eriksson, H. In-Depth Plasma Proteomics Reveals Increase in Circulating PD-1 during Anti-PD-1 Immunotherapy in Patients with Metastatic Cutaneous Melanoma. J. Immunother. Cancer 2020, 8 , e000204. [CrossRef] [PubMed]

97. Fejza, A.; Polano, M.; Camicia, L.; Poletto, E.; Carobolante, G.; Toffoli, G.; Mongiat, M.; Andreuzzi, E. The Efficacy of Anti-PD-L1 Treatment in Melanoma Is Associated with the Expression of the ECM Molecule EMILIN2. Int. J. Mol. Sci. 2021, $22,7511$. [CrossRef]

98. Wang, H.; Pan, J.; Barsky, L.; Jacob, J.C.; Zheng, Y.; Gao, C.; Wang, S.; Zhu, W.; Sun, H.; Lu, L.; et al. Characteristics of Pre-Metastatic Niche: The Landscape of Molecular and Cellular Pathways. Mol. Biomed. 2021, 2, 3. [CrossRef]

99. Ostrowski, S.M.; Fisher, D.E. Biology of Melanoma. Hematol. Oncol. Clin. N. Am. 2021, 35, 29-56. [CrossRef]

100. Van Allen, E.M.; Wagle, N.; Sucker, A.; Treacy, D.J.; Johannessen, C.M.; Goetz, E.M.; Place, C.S.; Taylor-Weiner, A.; Whittaker, S.; Kryukov, G.V.; et al. The Genetic Landscape of Clinical Resistance to RAF Inhibition in Metastatic Melanoma. Cancer Discov. 2014, 4, 94-109. [CrossRef]

101. Johnson, D.B.; Menzies, A.M.; Zimmer, L.; Eroglu, Z.; Ye, F.; Zhao, S.; Rizos, H.; Sucker, A.; Scolyer, R.A.; Gutzmer, R.; et al. Acquired BRAF Inhibitor Resistance: A Multicenter Meta-Analysis of the Spectrum and Frequencies, Clinical Behaviour, and Phenotypic Associations of Resistance Mechanisms. Eur. J. Cancer 2015, 51, 2792-2799. [CrossRef]

102. Rizos, H.; Menzies, A.M.; Pupo, G.M.; Carlino, M.S.; Fung, C.; Hyman, J.; Haydu, L.E.; Mijatov, B.; Becker, T.M.; Boyd, S.C.; et al. BRAF Inhibitor Resistance Mechanisms in Metastatic Melanoma: Spectrum and Clinical Impact. Clin. Cancer Res. 2014, 20, 1965-1977. [CrossRef]

103. Teh, J.L.F.; Cheng, P.F.; Purwin, T.J.; Nikbakht, N.; Patel, P.; Chervoneva, I.; Ertel, A.; Fortina, P.M.; Kleiber, I.; HooKim, K.; et al. In Vivo E2F Reporting Reveals Efficacious Schedules of MEK1/2-CDK4/6 Targeting and MTOR-S6 Resistance Mechanisms. Cancer Discov. 2018, 8, 568-581. [CrossRef]

104. Apostoli, P.; Minoia, C.; Hamilton, E.I. Significance and Utility of Reference Values in Occupational Medicine. Sci. Total Environ. 1998, 209, 69-77. [CrossRef]

105. Goel, V.K.; Lazar, A.J.F.; Warneke, C.L.; Redston, M.S.; Haluska, F.G. Examination of Mutations in BRAF, NRAS, and PTEN in Primary Cutaneous Melanoma. J. Investig. Dermatol. 2006, 126, 154-160. [CrossRef]

106. Tsao, H.; Goel, V.; Wu, H.; Yang, G.; Haluska, F.G. Genetic Interaction between NRAS and BRAF Mutations and PTEN/MMAC1 Inactivation in Melanoma. J. Investig. Dermatol. 2004, 122, 337-341. [CrossRef]

107. Mirmohammadsadegh, A.; Marini, A.; Nambiar, S.; Hassan, M.; Tannapfel, A.; Ruzicka, T.; Hengge, U.R. Epigenetic Silencing of the PTEN Gene in Melanoma. Cancer Res. 2006, 66, 6546-6552. [CrossRef]

108. Shi, H.; Hugo, W.; Kong, X.; Hong, A.; Koya, R.C.; Moriceau, G.; Chodon, T.; Guo, R.; Johnson, D.B.; Dahlman, K.B.; et al. Acquired Resistance and Clonal Evolution in Melanoma during BRAF Inhibitor Therapy. Cancer Discov. 2014, 4, 80-93. [CrossRef] 